Research Article

\title{
Shenjinhuoxue Mixture Attenuates Inflammation, Pain, and Cartilage Degeneration by Inhibiting TLR-4 and NF- $\kappa$ B Activation in Rats with Osteoarthritis: A Synergistic Combination of Multitarget Active Phytochemicals
}

\author{
Xiaoqin Ma $\mathbb{D}^{1,2}$ Chenxia Hao ${ }^{\mathbb{D}}{ }^{1,3}$ Zhaokang Zhang $\mathbb{D}^{1}$, Huiting Jiang $\mathbb{D}$, \\ Weixia Zhang $\mathbb{D},{ }^{1}$ Jingjing Huang $\mathbb{D},{ }^{1}$ Xiaofei Chen $\mathbb{D},{ }^{4}$ and Wanhua Yang $\mathbb{D}^{1}$ \\ ${ }^{1}$ Department of Pharmacy, Ruijin Hospital, Shanghai Jiao Tong University School of Medicine, Shanghai, China \\ ${ }^{2}$ Department of Pharmacy, Xi'an Children's Hospital, Xi'an, China \\ ${ }^{3}$ Department of Pharmacy, Shanghai Children's Medical Center, Shanghai Jiao Tong University School of Medicine, \\ Shanghai, China \\ ${ }^{4}$ School of Pharmacy, Second Military Medical University, Shanghai, China
}

Correspondence should be addressed to Jingjing Huang; hjj40694@rjh.com.cn, Xiaofei Chen; xfchen2010@163.com, and Wanhua Yang; yangwanhuaxy@163.com

Xiaoqin Ma and Chenxia Hao contributed equally to this work.

Received 16 May 2021; Revised 23 September 2021; Accepted 28 September 2021; Published 21 October 2021

Academic Editor: Raul Dominguez Perles

Copyright (c) 2021 Xiaoqin Ma et al. This is an open access article distributed under the Creative Commons Attribution License, which permits unrestricted use, distribution, and reproduction in any medium, provided the original work is properly cited.

Osteoarthritis (OA), a highly prevalent chronic joint disease, involves a complex network of inflammatory mediators that not only triggers pain and cartilage degeneration but also accelerates disease progression. Traditional Chinese medicinal shenjinhuoxue mixture (SHM) shows anti-inflammatory and analgesic effects against OA with remarkable clinical efficacy. This study explored the mechanism underlying anti-OA properties of SHM and evaluated its efficacy and safety via in vivo experiments. Through network pharmacology and published literature, we identified the key active phytochemicals in SHM, including $\beta$-sitosterol, oleanolic acid, licochalcone A, quercetin, isorhamnetin, kaempferol, morusin, lupeol, and pinocembrin; the pivotal targets of which are TLR- 4 and NF- $\kappa$ B, eliciting anti-OA activity. These phytochemicals can enter the active pockets of TLR- 4 and NF- $\kappa$ B with docking score $\leq-3.86 \mathrm{kcal} / \mathrm{mol}$, as shown in molecular docking models. By using surface plasmon resonance assay, licochalcone A and oleanolic acid were found to have good TLR-4-binding affinity. In OA rats, oral SHM at mid and high doses $(8.72 \mathrm{~g} / \mathrm{kg}$ and $26.2 \mathrm{~g} / \mathrm{kg})$ over 6 weeks significantly alleviated mechanical and thermal hyperalgesia $(P<0.0001)$. Accordingly, the expression of inflammatory mediators (TLR-4, interleukin (IL-) 1 receptor-associated kinase 1 (IRAK1), NF- $\kappa \mathrm{B}$ p65, tumor necrosis factor (TNF-) $\alpha$, IL-6, and IL- $1 \beta$ ), receptor activator of the NF- $\kappa$ B ligand (RANKL), and transient receptor potential vanilloid 1 (TRPV1) in the synovial and cartilage tissue of OA rats was significantly decreased $(P<0.05)$. Moreover, pathological observation illustrated amelioration of cartilage degeneration and joint injury. In chronic toxicity experiment of rats, SHM at $60 \mathrm{mg} / \mathrm{kg}$ demonstrated the safety. SHM had an anti-inflammatory effect through a synergistic combination of active phytochemicals to attenuate pain and cartilage degeneration by inhibiting TLR- 4 and NF- $\kappa$ B activation. This study provided the experimental foundation for the development of SHM into a more effective dosage form or new drugs for OA treatment.

\section{Introduction}

Osteoarthritis $(\mathrm{OA})$ is the most prevalent joint disease associated with old age, which is affecting around 240 million people worldwide [1]. In elderly people, OA is a leading cause of disability and significantly increases the social and economic burden [2]. OA is primarily attributed to chronic inflammation, which is responsible for multiple $\mathrm{OA}$ 
phenotypes [3]. In OA, inflammation is triggered and developed by an intrinsic interaction between local tissue damage or metabolic dysfunction products, called damage-associated molecular patterns and the innate immune system via pattern-recognition receptors (PRRs) [4].

As an important PRR, Toll-like receptor (TLR-) 4 is widely expressed in chondrocytes and synovial macrophages. During OA, TLR-4 activation triggers the nuclear factor kappa B (NF$\kappa \mathrm{B})$ pathway with the secretion of inflammatory cytokines and chemokines such as tumor necrosis factor (TNF-) $\alpha$ and interleukin (IL-) 6, resulting in pain and cartilage degradation [5, 6]. Furthermore, TLR- 4 can mediate the classically activated type 1 (M1) polarization of macrophages, worsening the inflammatory response in OA [7]. Then, a plethora of inflammatory cytokines, such as TNF- $\alpha$, stimulates the expression of the receptor activator of the NF- $\kappa \mathrm{B}$ (RANK) ligand (RANKL) on osteoblasts [8]. Study [9] demonstrated that the RANKL/RANK pathway regulates osteochondral crosstalk, involved in OA progression. TNF- $\alpha$ also activates transient receptor potential vanilloid 1 (TRPV1) that is an important sensor on peripheral nerve endings and nonneuronal synoviocytes in the knee joint [10]. TRPV1 functions as a molecular integrator of nociceptive stimuli such as thermal and mechanical stimuli abundant in inflamed joints, thus regulating pain and inflammation [11]. Owing to the complex network of inflammatory mediators and multifactorial etiology in OA, diseasemodifying OA drugs (DMOADs) targeting a single inflammatory mediator, such as strontium ranelate, an IL- $1 \beta$ inhibitor, demonstrated unsatisfactory efficacy with diverse adverse effects. DMOADs that hit multiple inflammatory targets might have great therapeutic potential [12].

Herbal medicines and herb-derived active phytochemicals have recently been novel approaches with great potential for OA therapy, attributing to their chondroprotective and osteoprotective properties [13-15]. For OA treatment, traditional Chinese medicine (TCM), showing effective efficacy with safety, has been gaining more and more interest and recommended in combination with Western medicine by the pharmacologic guidelines of China [16, 17]. Furthermore, compared to Western medicine, TCM treatment significantly increased the total effective rate and decreased the recurrence rate in OA patients $(P<0.00001)$ [18]. TCM, characterized by "holistic therapy," has multiple phytochemicals that hit multiple targets involved in inflammation of OA, joint activity function, and bone metabolism [19]. By combining the ingredients relying on the "principal (Jun), minister (Chen), assistant (Zuo), and guide (Shi)", a compound prescription of TCM can yield a synergistic effect for treating diseases via the action on multiple targets and pathways [20]. Shenjinhuoxue mixture (SHM) is derived from "Wei's traumatology" that is a famous Chinese medical sect and selected as an intangible cultural heritage. SHM has been approved by Shanghai Food and Drug Administration to use in OA patients at Ruijin Hospital for decades and provided a good curative effect with safety.

In a previous study, we predicted that SHM had antiinflammatory and analgesic actions against OA through multiple active phytochemicals with high oral bioavailability $(\mathrm{OB})(\mathrm{OB}>30 \%)$ and drug likeness $(\mathrm{DL})(\mathrm{DL}>0.18)[21]$.
These are the criteria for bioactive phytochemicals commonly used in many studies. However, pharmacological activity of some phytochemicals with low OB may be underestimated, such as ursolic acid $(\mathrm{OB}=16.77 \%)$, because of the enhanced bioactivity through interplays with gut microbiota. In addition, more and more data, including pharmacokinetic (PK) profiles, regarding the anti-OA properties of active phytochemicals in SHM have been reported. Therefore, this study explored further the mechanism underlying synergistic anti-OA properties of SHM and evaluated its pharmacological action and safety in in vivo experiments. The flowchart is shown in Figure 1.

\section{Materials and Methods}

\subsection{Predicting Mechanism of SHM against OA}

2.1.1. Dissection of SHM against OA by Network Pharmacology Analysis and Published Literatures. (1) Screening of Active Phytochemicals in Herbs of SHM against OA

The SHM formula is composed by 12 kinds of herbs as follows: Lycopodii Herba $(L H)$ as the principal; Radix Angelicae Sinensis (RAS), Radix Paeoniae Alba (RPA), Carica papaya L. (CPL), Frankincense (FK), Myrrha $(M H)$, and Radix Gentianae Macrophyllae (RGM) as the minister; Visci Herba (VH), Cibot Rhizome (CR), and Radix Dipsaci (RD) as the assistant; and Radix Cyathulae $(R C)$ and Radix Glycyrrhizae $(R G)$ as the guide.

The phytochemicals in SHM herbs active in OA therapy were those present in herbs of SHM as well as those related with OA therapy. First, all the phytochemicals were screened from the traditional Chinese medicine system pharmacology (TCMSP) database (http://lsp.nwu.edu.cn/browse.php) and supplemented by literature from PudMed/MEDLINE, ScienceDirect, Spring Link, Web of Science, CNKI, and VIP database from 2014 to 2020 . The active phytochemicals were those with anti-OA activities reported in in vitro or in vivo experiments. High-frequency shared active phytochemicals were identified as the key active phytochemicals based on their possible high levels in SHM.

(2) Identification of Active Phytochemical-Related Targets of SHM against OA, Herb-Active Phytochemical-Target Network Construction, and Kyoto Encyclopedia of Genes and Genomes Pathway Database Pathway Enrichment Analysis

The active phytochemical-related targets of SHM against OA were the shared part of the active phytochemical-related targets and the OA-related targets. The targets related to the active phytochemicals in SHM were identified through chemical similarities and pharmacophore models in the SwissTargetPrediction database (http://www.swisstargetprediction.ch). The OA-related targets were collected from the Online Mendelian Inheritance in Man database (http://www.omim.org/ ,download), the Kyoto Encyclopedia of Genes and Genomes Pathway (KEGG) database http://www.kegg.jp/,download), DisGeNET database (https://www.disgenet.org/search), and DrugBank database (https://www.drugbank.ca/). The proteinprotein interactions (PPI) of each active phytochemicalrelated target against OA were generated using the STRING database. The targets having the interactions with a 


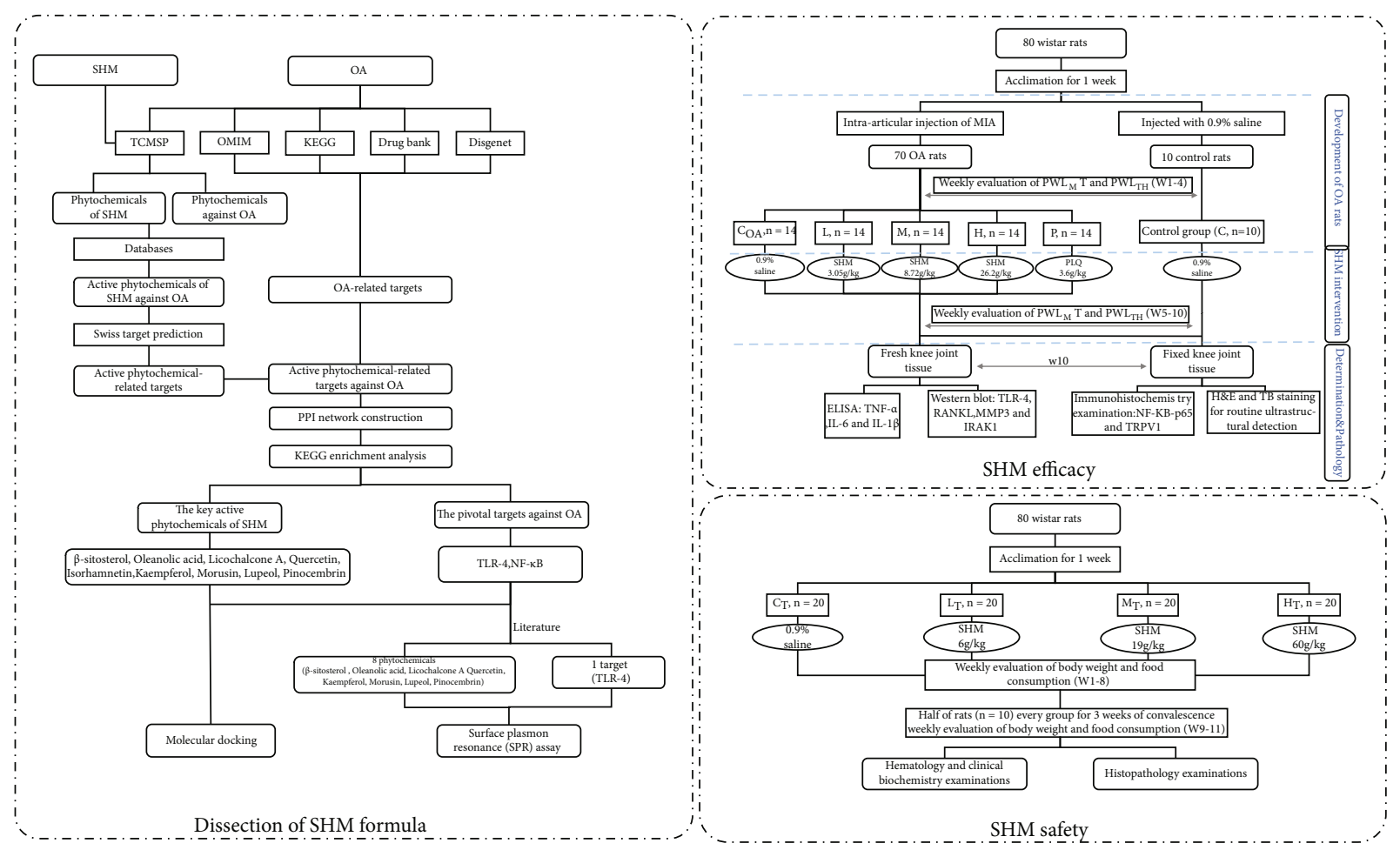

FIgURE 1: Flowchart of this study.

probabilistic association confidence score of $\geq 0.9$ were selected as the potential targets for further network construction in this study [22]. Network topology parameters (e.g., median and maximum degrees of freedom) in the tab-separated values (TSV) data format using the network analyzer in Cytoscape (version 3.7.1) we used. The key targets with degree values exceeding the mean degree value of the entire network, subjected to KEGG signaling pathway enrichment analysis using the $\mathrm{R}$ project, were used. The pathways with $P$ of $<0.05$ were considered statistically significant.

To elucidate the relationship among the herbs, active phytochemicals, and potential targets, the herb-active phytochemical-target network was constructed. Then, these phytochemicals with the highest degree value were also included as the key active phytochemicals. Moreover, the pivotal targets in SHM were those playing a crucial regulatory role in OA treatment.

(3) Key Active Phytochemical-Target Molecular Docking

The 2D structures of the key active phytochemicals were downloaded from PubChem database (https://pubchem .ncbi.nlm.nih.gov/). The $2 \mathrm{D}$ structure was transferred to a $3 \mathrm{D}$ chemical structure to minimize energy for further docking. The 3D structures of the protein targets were downloaded from Protein Data Bank (https://www.rcsb.org/pages/ contactus). The proteins were embellished by PyMOL (https://pymol.org/) to remove the original ligand, water molecules, and phosphates. Furthermore, the receptors were prepared by the AutoDockTools (version 1.5.6: http:// mgltools.scripps.edu/documentation/links/autodock), including hydrogen addition and docking parameter settings. "Grid box" was set to maximum to perform the blind docking. All ligand and receptor files were saved as pdbqt format.
Finally, AutoDock Vina was used to evaluate and verify the binding affinity of the ligand-receptor relationship. For each ligand, the lowest binding energy was selected as the result of molecular docking. The docking conformation analysis and mapping were performed using PyMOL software, and PLIP (https://projects.biotec.tu-dresden.de/ plip-web/plip) was used to analyze the force between ligands and proteins. In general, it is believed that the binding capacity is stronger when the dock binding-free energy is $<-4 \mathrm{kcal} / \mathrm{mol}$. The molecular docking model was considered accurate, reliable, and reliable or accurate if its root mean square deviation from the crystal structure was $\leq 2, \leq 4$, and $<3 \AA$, respectively [23].

2.1.2. Analysis of Interactions between Active Phytochemicals and Protein Targets by Surface Plasmon Resonance Assay. Surface plasmon resonance (SPR) measurements were performed using a Biacore T200 instrument (GE Healthcare, Uppsala, Sweden). The protein was immobilized on a CM5 sensor chip via the primary amine groups. Before use, the CM5 sensor chip was activated by using sulfo-NHS/EDC chemistry in a buffer (composed of $2.7 \mathrm{mM} \mathrm{KCl,} 137 \mathrm{mM}$ $\mathrm{NaCl}, 0.05 \%(v / v)$ surfactant P20, pH 7.4; chemicals and regents were listed in file of supplementary materials). The affinity of key phytochemicals to the protein targets was assessed using a Biacore T200 Evaluation Software (version 3.0). The key phytochemicals were flowed at a rate of $30 \mathrm{~L} / \mathrm{min}$ for $60-180 \mathrm{~s}$ to allow for association, followed by another $300 \mathrm{~s}$ for dissociation, over immobilized protein in $\mathrm{PBS} / 5 \%$ DMSO running buffer $(1.05 \times \mathrm{PBS}, 0.5 \% \mathrm{P} 20$ surfactant, 5\% DMSO, pH 7.4). The phytochemicals were tested for binding at $3.125 \mu \mathrm{M}$ to $200 \mu \mathrm{M}$. Data normalization 
involved transformation of the $y$-axis such that the theoretical maximum amount of binding for a $1: 1$ interaction with the protein surface corresponded to a sensor response of 100 relative units (RUs).The bound ability of small molecules to the target was evaluated by equilibrium dissociation constant $\left(K_{\mathrm{D}}\right)[24]$.

\subsection{Evaluating the Pharmacological Effects of SHM on the OA Rat Model and Its Potential Chronic Toxicity In Vivo}

2.2.1. Chemicals, Reagents, and Animals. SHM was obtained from Ruijin Hospital, Shanghai Jiaotong University School of Medicine (Shanghai, China), and its preparation method with the quality standard was listed in the file of supplementary materials. Panlongqi tablets (PLQ) were purchased from Panlong Pharmaceutical Group Limited by Share Ltd. (Shaanxi, China), and monosodium iodoacetate (MIA) was purchased from Macklin Biochemical Technology Co. Ltd. (Shanghai, China). Rat ELISA assay kits of TNF- $\alpha$, IL-6, and IL- $1 \beta$ were obtained from Nanjing Jiancheng Bioengineering Institute (Nanjing, China). Anti-TLR-4 rabbit monoclonal antibody, anti-matrix metalloproteinase 3 (anti-MMP3) rabbit monoclonal antibody, and anti-actin mouse monoclonal antibody were bought from Servicebio Technology Company (Wuhan, China). Anti-RANKL rabbit monoclonal antibody was obtained from Boster (Wuhan, China), and anti-IL-1 receptor-associated kinase 1 (IRAK1) rabbit monoclonal antibody was purchased from Proteintech (Wuhan, China). Secondary antibody anti-rabbit HRP, anti-mouse HRP, 5x protein loading buffer, SDS-PAGE gel preparation kit, 5\% skim milk, and ECL solution were obtained from Servicebio (Wuhan, China). Reagent absolute ethanol, xylene, hydrochloric acid, ammonia, and neutral gum were obtained from Sinopharm Chemical Reagent Co. Ltd. (Shanghai, China). Hematoxylin-eosin dye (HE) and toluidine blue (TB) were bought from Servicebio (Wuhan, China), and efficient sectioning paraffin was purchased from Shanghai Huayong Olefin Co. Ltd. (Shanghai, China).

In total, 160 healthy-specific pathogen-free Wistar rats aged 8 weeks and weighing 180-200 g were purchased from Xipuer-Beikai Experimental Animal Co. Ltd. (Shanghai, China). All rats were housed in ventilated racks at $24 \pm 2{ }^{\circ} \mathrm{C}$ with relative humidity at $45 \% \pm 5 \%$ on a $12 \mathrm{~h}$ light/dark cycle (light on from 7:00 to 19:00). The rats were permitted to acclimate to laboratory conditions at least 1 week before the start of experiments. Animals in pairs were maintained in the cages that were lined with woodchip bedding, and animals were provided with environmental enrichment. Standard solid pellet feed and fresh water were provided ad libitum. All 160 rats were equally divided into pharmacological and chronic toxicity experiments. The experimental protocols were reviewed and approved by the ethics committee of the Shanghai Institute of Pharmaceutical Industry (no. 2019-0051-1 and 2019-0051-2).

2.2.2. Pharmacological Experiment for SHM Effects. Seventy rats were given the intraarticular injection of MIA (for a single dose, $2 \mathrm{mg}$; dissolved in $50 \mu \mathrm{L}$ of $0.9 \%$ saline) in the right posterior knee to induce the OA model [25]. Then, the knees of rats were bent and straightened alternately for $30 \mathrm{~s}$ to disperse MIA throughout the joint. The other 10 rats were intragastrically injected with $0.9 \%$ saline as the control (C) group. Subsequently, the mechanical hyperalgesia $(\mathrm{MH})$ and thermal hyperalgesia (TH) of all rats were analyzed weekly to evaluate the rat knee OA models. Rats with significantly lower $\mathrm{MH}$ and $\mathrm{TH}$ than the $\mathrm{C}$ group $(P<0.05)$ for 4 consecutive weeks (W1-4) were considered as osteoarthritic.

All OA rats were randomly divided into five groups on average as follows: (1) OA control $\left(\mathrm{C}_{\mathrm{OA}}\right),(2)$ low SHM intervention $(\mathrm{L},(3.05 \mathrm{~g} / \mathrm{kg}) 1.4 \mathrm{~mL} / \mathrm{kg})$, (3) mid SHM intervention $(\mathrm{M},(8.72 \mathrm{~g} / \mathrm{kg}) 4 \mathrm{~mL} / \mathrm{kg})$, (4) high SHM intervention $(\mathrm{H},(26.2 \mathrm{~g} / \mathrm{kg}) 12 \mathrm{~mL} / \mathrm{kg})$, and (5) positive control with (P: PLQ, 3.6 g/kg). SHM, PLQ, and $0.9 \%$ saline were orally administered once daily for 6 consecutive weeks (W5-10). The mid-SHM dosage of the rat was approximately 6 times the daily dosage of human [26], and calculations of low-, mid-, and high-SHM dosages of rats were listed in the file of supplementary materials. High- and low-SHM dosages were three times and a third of the mid dosage, respectively [27]. In addition, the 10 rats of the $C$ group were administered $0.9 \%$ saline orally.

(1) Analgesia Evaluation by $\mathrm{MH}$ and TH Tests

The $\mathrm{MH}$ and $\mathrm{TH}$ of $\mathrm{OA}$ and $\mathrm{C}$ rats were tested weekly during the intervention phase (W5-10). $\mathrm{MH}$ and $\mathrm{TH}$ were assessed using the electronic Von Frey test (IITC, USA) and thermal plantar tester (IITC, USA), respectively. The electronic Von Frey test was used to automatically record the mechanical force applied to the tip of the device through a manageable force transducer and the paw withdrawal threshold $\left(\mathrm{PWL}_{\mathrm{MH}}\right)$ displayed on the screen. The thermal plantar tester automatically deactivated the radiant heat source when the animal withdrew its paw, and the latency was recorded as $\mathrm{PWL}_{\mathrm{TH}}$. These tests were repeated three times to calculate the average values of PWL in response to the mechanical and thermal forces. In addition, each hind paw was tested with a cutoff time of $20 \mathrm{~s}$ during each immersion so as to prevent tissue damage if a rat failed to withdraw its paw.

(2) Determination of TLR-4, IRAK1, TNF- $\alpha, I L-6, I L-1 \beta$, RANKL, and MMP3 Levels in Synovial and Cartilage Tissues of $O A$ Rats

At the end of the experiments (W10), fresh synovial and cartilage tissues were extracted from the posterior knee joints of $\mathrm{OA}$ rats and stored at $-80^{\circ} \mathrm{C}$. All the $\mathrm{OA}$ rats were euthanatized before tissue extraction. Then, half of the tissue block of every OA rat was washed with PBS two to three times, cut into small pieces, placed in homogenization tube with one or two small magnetic beads $(2 \mathrm{~mm})$, and lysed in 10 times volume of tissue buffer by adding protease inhibitors. The homogenization tube was shaken for $30 \mathrm{~min}$ in an ice bath and then centrifuged at $12000 \mathrm{rpm}$ ( $1 \mathrm{~min}$ ). Finally, the supernatant, which was the total protein solution, was collected for further analysis.

TNF- $\alpha$, IL- $1 \beta$, and IL- 6 levels in the synovial and cartilage tissues of rats were determined using the rat-specific ELISA kits according to the manufacturer's instructions. TLR-4, IRAK1, RANKL, and MMP3 protein levels were measured through Western blot analysis. In brief, total 
protein was denatured $5 \times$ protein loading buffer in a boiling water bath. Equal amounts of total protein were electrophoresed on a $10 \%$ acrylamide SDS gel; they were then electrotransferred onto a PVDF membrane and activated using methanol at a constant voltage of $25 \mathrm{~V}$. After being blocked with skimmed milk powder for $2 \mathrm{~h}$ at room temperature, the membranes were incubated at $4^{\circ} \mathrm{C}$ overnight with antibody specific for the target protein (listed in supplementary materials) and then $1 \mathrm{~h}$ with the corresponding secondary antibody $(1: 3000)$. The membranes were washed three times with TBST and detected with EGL plus kit in a Western blotting detection system. The density of each target band was quantified using Alpha software (Alpha Innotech, USA) and normalized to $\beta$-actin via optical density.

(3) Pathological Observation of Synovial Fluid and Cartilage in OA Rats

The other half of synovial and cartilage tissue was fixed in $25 \%$ glutaraldehyde solution for $48 \mathrm{~h}$. Fixed tissues were processed for paraffin embedding, and 3-5 $\mu \mathrm{m}$ thick sections were prepared for immunohistochemical, ultrastructural, and histopathological examination. Immunohistochemistry examination was used to detect NF- $\kappa$ Bp65 and TRPV1 content and distribution. Then, the density of the brown color developed because immunostaining was measured and then statistically analyzed. All sections were examined using a light microscope (Olympus, Tokyo, Japan) with an attached charge-coupled device digital camera (Mingmei Shot 60).

Sample sections were stained with hematoxylin and eosin (H\&E) and TB for routine ultrastructural and histopathological examination, respectively, followed by morphometric analysis, at 200x magnification.

2.2.3. Chronic Toxicity Experiment for SHM Safety. Eighty rats were randomly divided into four groups $(n=20,10$ males and 10 females) for chronic toxicity analysis as follows: (1) normal control $\left(\mathrm{C}_{\mathrm{T}}\right)$, (2) low-dose SHM toxicity $\left(\mathrm{L}_{\mathrm{T}}, 6 \mathrm{~g} / \mathrm{kg}\right)$, (3) mid-dose SHM toxicity $\left(\mathrm{M}_{\mathrm{T}}, 19 \mathrm{~g} / \mathrm{kg}\right)$, and (4) high-dose SHM toxicity $\left(\mathrm{H}_{\mathrm{T}}, 60 \mathrm{~g} / \mathrm{kg}\right)$.

Before administration, SHM was condensed into an extractum ( $1 \mathrm{~g}$ of extractum $=9.6 \mathrm{~g}$ of dried medicinal herbs) to achieve a final dilution which was $100 \mathrm{~g} / 1 \mathrm{~mL}$. Here, $0.9 \%$ saline and SHM at different doses were given orally to the $\mathrm{C}_{\mathrm{T}}$ and other groups, respectively, once daily for 8 consecutive weeks.

At the end of the eighth week, half of the rats $(n=10$, five male and five female) of each group, were euthanatized and whole blood samples were taken from their abdominal aorta for the tests of hematology and clinical biochemistry testing. All organs were weighed before histopathological examinations.

After another 3 weeks, we check the metabolism and elimination of SHM (convalescence). Here, the other 10 rats of each group were maintained and then necropsied after euthanasia for toxicity examination.

(1) Clinical Observations, Body Weight, and Food Consumption

All animals in the chronic toxicity experiment were observed twice daily for clinical manifestation of toxicity. In brief, they were checked for changes in the skin, fur, eyes, and mucous membranes. Their mean body weight and food consumption were calculated weekly for each rat individually throughout the testing period.

(2) Hematology, Blood Coagulation, and Clinical Biochemistry

Hematologic assessments were performed on an automated hematology analyzer (MEK-6318, Nihon Kohden, Tokyo, Japan), and the results included white blood cell count, red blood cell count, hemoglobin concentration $(\mathrm{Hb})$, hematocrit (HCT), neutrophilic granulocyte percent (NE), lymphocyte percent (LY), monocyte percent (MO), and platelet count (PLT). Serum biochemical parameters, including alanine aminotransferase (ALT), total protein (TP), albumin (ALB), total bilirubin (TBIL), aspartate aminotransferase (AST), total cholesterol $(\mathrm{CHO})$, blood urea nitrogen $(\mathrm{BUN})$, creatinine $(\mathrm{Cr})$, glucose (GLU), sodium $(\mathrm{Na})$, triglycerides $(\mathrm{TG})$, potassium $(\mathrm{K})$, and chloride $(\mathrm{Cl})$, were evaluated using an automated biochemical analyzer (7020, Hitachi, Tokyo, Japan). Prothrombin time (PT) and activated partial thromboplastin time (APTT) for blood coagulation were analyzed on a blood coagulation analyzer (CA-530, Sysmex, Kobe, Japan).

(3) Histopathology

The heart, liver, spleen, lungs, kidneys, adrenal glands, brain, thymus, and testes of each rat were weighed. Relative organ weights were calculated as relative organ weight $(\%)=$ [organ weight $(\mathrm{g}) /$ body weight $(\mathrm{g})] \times 100$.

The peripheral oral cavity, cranial cavity, and all tissues and organs in the thoracic and abdominal cavity were examined visually for any abnormalities. The organs and tissues were fixed in 10\% neutral-buffered formalin, embedded in paraffin, sectioned at 3-5 $\mathrm{m}$ thickness, and stained with $\mathrm{H} \& \mathrm{E}$ for microscopic examination. All sections were observed under an optical microscope (Olympus).

2.3. Statistical Analysis. All animal data from pharmacologic and toxicity experiments were expressed as means \pm SEMs and means \pm SDs, respectively. Data of two groups were compared using the parametric Student's $t$-test or the nonparametric Mann-Whitney test. Comparison among three or more groups was performed using one-way analysis of variance, followed by Tukey's post hoc test. All data were analyzed using SPSS for Windows (version 24; IBM, Chicago, IL, USA). The graphs were plotted using GraphPad Prism (version 8; GraphPad, San Diego, CA, USA). $P<0.05$ was considered statistically significant.

\section{Results}

\subsection{Anti-Inflammatory Effects of SHM against OA via the Inhibition of TLR-4 and NF- $\kappa B$ Activation}

3.1.1. Identification of the Nine Key Active Phytochemicals of SHM and the Key Targets against Inflammation of OA. In total, 327 phytochemicals in herbs of SHM against OA were obtained from TCMSP database and supplemented by literatures after eliminating the duplicates. There were 67 active phytochemicals, including three supplemented alkaloids ( $\alpha$-obscurine, lycojaponicumin C, and lycodoline) of 
LH [28] and one phytochemical (kojic acid) of CR. Distribution of active phytochemicals among the herbs of the principal, minister, assistant, and guide in SHM is shown in Figure 2(a). The key active phytochemicals of $\beta$-sitosterol and oleanolic acid were found in seven herbs (RAS, RPA, $M H, R G M, V H, R D$, and $R C)$ and six herbs (RPA, RGM, $C P L, V H, R C$, and $R G$ ), respectively.

Totally, 249 active phytochemical-related targets against OA were the shared part of 573 active phytochemical-related targets and 825 OA-related targets (Figure 2(b), Supplementary Tables). The PPI network is composed of 173 potential targets and 2699 interactions with an average degree value of 31.2. The 63 key targets (the degree value $>31.2$ ) have 1235 interactions, including the top 10: IL-6, TNF, VEGFA, TLR4, IL-10, CXCL8, AKT1, IL-1 $\beta$, and NF- $\kappa$ B1 (Figure 2(c)). The KEGG analysis results showed that these key targets totally contributed to 98 pathways ( $P$ value $<0.05$ ).

The top 10 pathways included the TLR signaling pathway, TNF signaling pathway, and NF- $\kappa$ B signaling pathway which were closely correlated with the inflammation and cell death, as shown in Figure 2(d). The herb-active phytochemical-target network (Figure 2(e)) has 254 nodes and 2074 edges. In particular, the key targets of active phytochemicals in assistant and guide herbs included ILs, PIK3R1, and CYP3A4, indicating their anti-inflammation and anti-apoptotic effects and their effects on drug and toxin metabolism.

Based on this network, licochalcone A, quercetin, isorhamnetin, kaempferol, morusin, lupeol, and pinocembrin were also the key active phytochemicals with the highest degree value of 26.9. The results of published studies (Table 1) confirmed that the anti-OA properties of the nine key active phytochemicals involved the inhibition of TLR-4 and NF- $\kappa \mathrm{B}$ activation, accompanied by hepatoprotective and renoprotective effects. Thus, TLR- 4 and NF- $\kappa$ B were identified as the pivotal anti-OA targets for SHM and they were further analyzed.

3.1.2. Molecular Docking of the Key Active Phytochemicals and the Pivotal Targets of TLR-4 and NF- $\kappa B$. All these nine key active phytochemicals could easily enter and bind to the active pocket of the TLR- 4 and NF- $\kappa$ B protein as shown in Figure 3(a). The simulated results showed that the most binding complexes were lupeol-TLR-4 docking $(-7.03 \mathrm{kcal} / \mathrm{mol})$ and oleanolic acid-NF- $\kappa \mathrm{B}$ docking $(-8.52 \mathrm{kcal} / \mathrm{mol})$, as listed in Table 2. The hydrogen bond was the main form of interaction. The hydroxyl and carbonyl groups of the phytochemicals formed hydrogen bonds with the proteins.

Moreover, the interaction distances (root mean square deviations) between those phytochemicals and proteins were $<3.9 \AA$, representing the accuracy or reliability of the molecular docking models.

3.1.3. Binding Affinity between TLR-4 and the Phytochemicals of Licochalcone A and Oleanolic Acid. Isorhamnetin of the nine phytochemicals has been proven to block TLR-4 [55]. Thus, the other eight phytochemicals were selected as the candidate phytochemicals for SPR assay to clarify their interactions with TLR-4.
As shown in Figure 3(b), sodium acetate ( $\mathrm{pH} 5.0$ ) was selected as the dilution buffer to dilute TLR- 4 to $50 \mu \mathrm{g} / \mathrm{mL}$ in the immobilization assay because it obtained the highest bound response. Moreover, the bound response of the immobilization level was $3324.5 \mathrm{RU}$. The good affinity between lipopolysaccharide (LPS; positive reference standard) and TLR- 4 with a $K_{\mathrm{D}}$ of $3.41 \times 10^{-4}$ confirmed the activity of TLR-4. The results of the affinity assays showed that the $K_{\mathrm{D}}$ for the licochalcone A-TLR-4 and oleanolic acid-TLR-4 interaction was $7.80 \times 10^{-5}$ and $3.66 \times 10^{-4}$, respectively, indicating the affinity between these two phytochemicals and TLR-4 (Figure 3(c)).

\subsection{Pharmacological Effects of SHM against $O A$ and Its Safety in Rats}

3.2.1. Anti-Inflammatory and Analgesic Effects of SHM via Inhibiting TLR-4 and NF- $\kappa B$ Activation in OA Rats. (1) SHM Improved $\mathrm{MH}$ and $\mathrm{TH}$ of $\mathrm{OA}$ Rats

After a single dose of intraarticular injection with MIA, differences in the $\mathrm{PWL}_{\mathrm{MH}}$ and $\mathrm{PWL}_{\mathrm{TH}}$ values between the $\mathrm{OA}$ and $\mathrm{C}$ groups were significant on W2 $(P<0.05)$; the values gradually increased further on W4 $(34.2 \pm 3.14 \mathrm{~g}$ and $7.05 \pm 0.631 \mathrm{~s}$ for $\mathrm{C}_{\mathrm{OA}}$ vs. $80.0 \pm 1.80 \mathrm{~g}$ and $13.0 \pm 0.834 \mathrm{~s}$ for $\mathrm{C}$, respectively; $P<0.0001)$. These results indicated that our rat OA model was well established (Figure 4(a)).

SHM interventions increased the $\mathrm{PWL}_{\mathrm{MH}}$ and $\mathrm{PWL}_{\mathrm{TH}}$ in a time-dose-dependent manner (Figure 4(b)). At the end of the experiments (W10), compared with the $\mathrm{C}_{\mathrm{OA}}$ group, the groups treated with mid and high doses of SHM showed significant increases in both $\mathrm{PWL}_{\mathrm{MH}}(67.3 \pm 13.6$ and $76.7 \pm 8.43 \mathrm{~g}$, respectively) and $\mathrm{PWL}_{\mathrm{TH}}(10.9 \pm 0.245$ and $12.6 \pm 0.263 \mathrm{~s}$, respectively; all $P<0.0001)$. High-dose SHM showed the similar amelioration of $\mathrm{MH}$ and TH with PLQ (P group; $\mathrm{PWL}_{\mathrm{MH}}$ : $78.7 \pm 9.98 \mathrm{~g}, P=0.38$ and $\mathrm{PWL}_{\mathrm{TH}}: 11.8 \pm 0.261 \mathrm{~s}, P=0.73$; Figure 4(c)).

(2) Effect of SHM on TLR-4, RANKL, IRAK1, TNF- $\alpha$, IL-6, IL-1 $\beta$, and MMP3 Levels in the Synovial and Cartilage Tissue of OA Rats

As shown in Figures 5(a) and 5(b), compared with control rats, OA rats had higher TNF- $\alpha$, IL- $1 \beta$, and IL- 6 in the synovial and cartilage tissue (TNF- $\alpha: 4.28 \pm 0.791 \mathrm{ng} / \mathrm{L}$ (C) vs. $41.27 \pm 6.06 \mathrm{ng} / \mathrm{L}\left(\mathrm{C}_{\mathrm{OA}}\right), P<0.0001$; IL-1 $\beta$ : $79.0 \pm 7.57$ ng/L (C) vs. $216 \pm 6.38 \mathrm{ng} / \mathrm{L}\left(\mathrm{C}_{\mathrm{OA}}\right), P<0.0001$; and IL-6: $52.8 \pm 7.44 \mathrm{ng} / \mathrm{L}(\mathrm{C})$ vs. $\left.89.0 \pm 12.5 \mathrm{ng} / \mathrm{L}\left(\mathrm{C}_{\mathrm{OA}}\right), P=0.002\right)$. Notably, SHM interventions at mid and high doses showed the obvious anti-inflammatory effect by reducing the levels of aforementioned cytokines (TNF- $\alpha$ : $22.6 \pm 7.63 \mathrm{ng} / \mathrm{L}$ (mid) and $16.9 \pm 5.27 \mathrm{ng} / \mathrm{L}$ (high), $P<0.01$; IL-1 $\beta: 128 \pm$ $26.8 \mathrm{ng} / \mathrm{L}$ (mid) and $96.8 \pm 3.71 \mathrm{ng} / \mathrm{L}$ (high), $P<0.05$; and IL-6: $33.8 \pm 12.6 \mathrm{ng} / \mathrm{L}$ (mid), $28.9 \pm 3.50 \mathrm{ng} / \mathrm{L}$ (high), $P<$ 0.01 ). The effects were similar to those for PLQ (TNF- $\alpha$, $14.9 \pm 5.76 \mathrm{ng} / \mathrm{L} ; \mathrm{IL}-1 \beta, 100 \pm 13.8 \mathrm{ng} / \mathrm{L} ;$ and IL-6, $37.0 \pm$ $11.0 \mathrm{ng} / \mathrm{L}$ ) at the end of W10.

In line with these results, the Western blotting results indicated that increased expression of TLR-4, IRAK1, RANKL, and MMP3 was observed in OA rats $(P<0.01$, $P<0.01, P<0.0001$, and $P<0.05$, respectively). Moreover, in the synovial and cartilage tissue, high-dose SHM could 

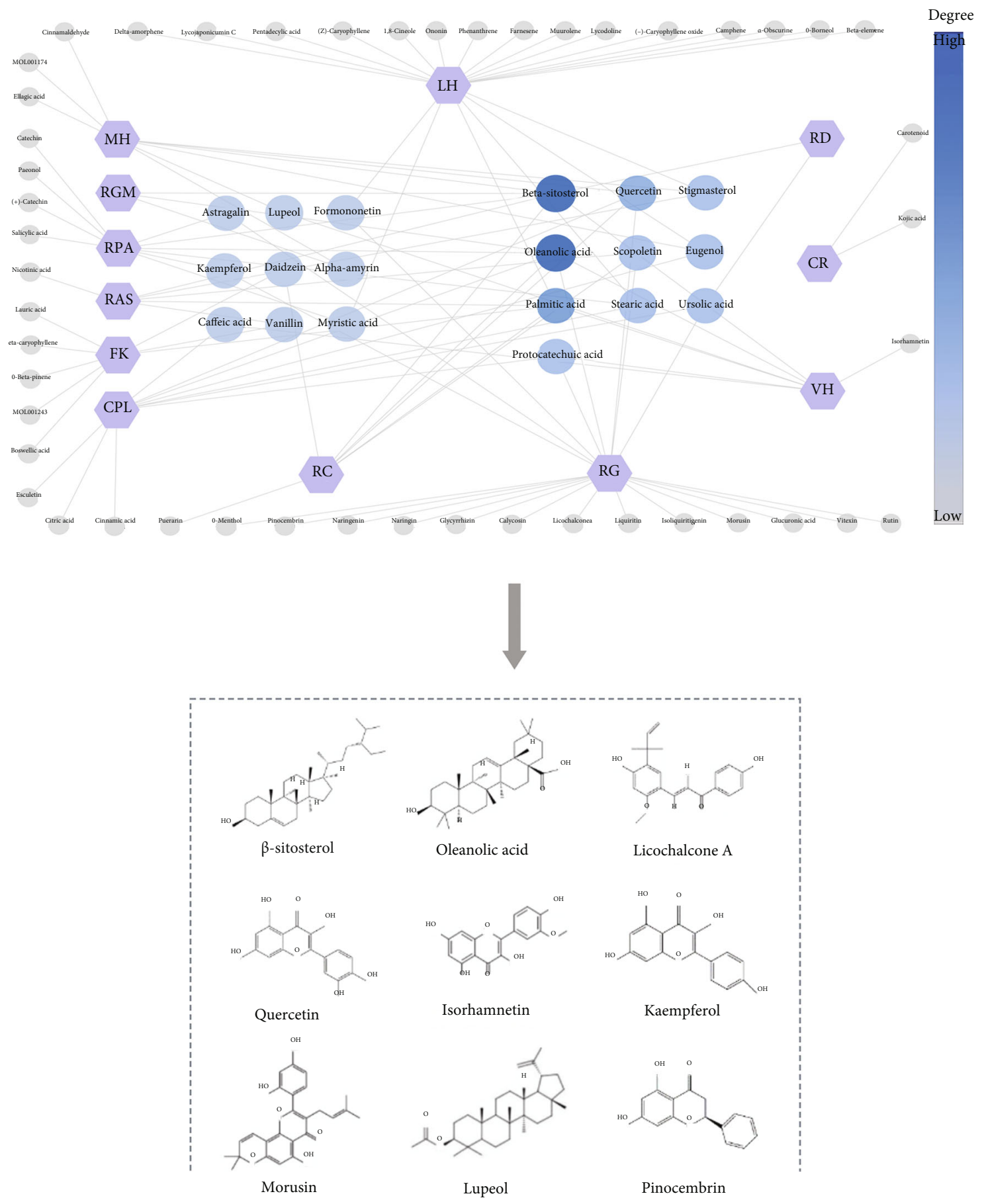

(a)

Figure 2: Continued. 


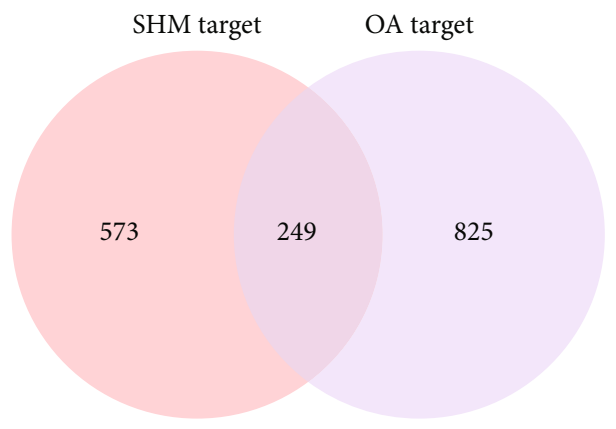

(b)

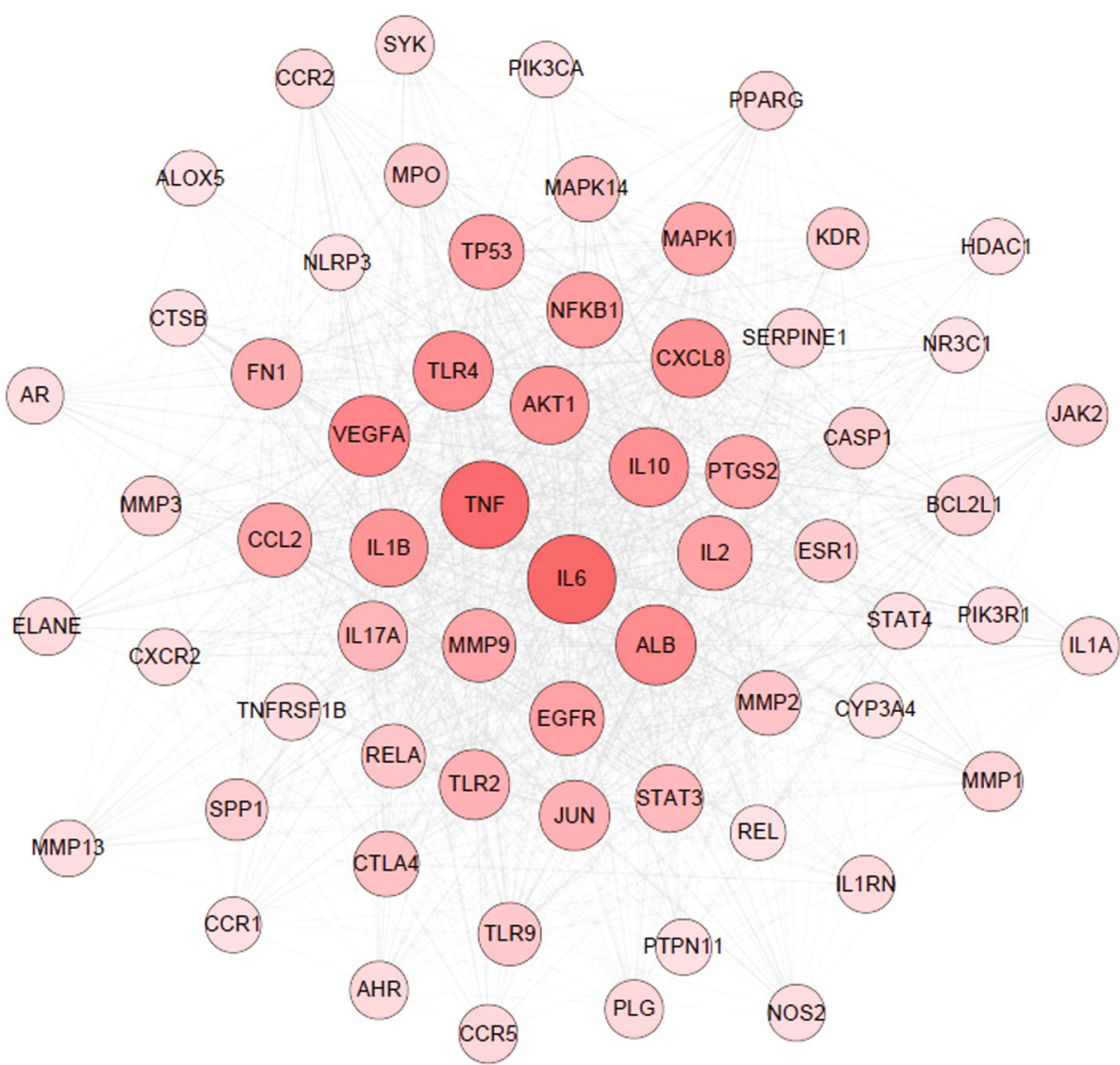

(c)

FIgUre 2: Continued. 


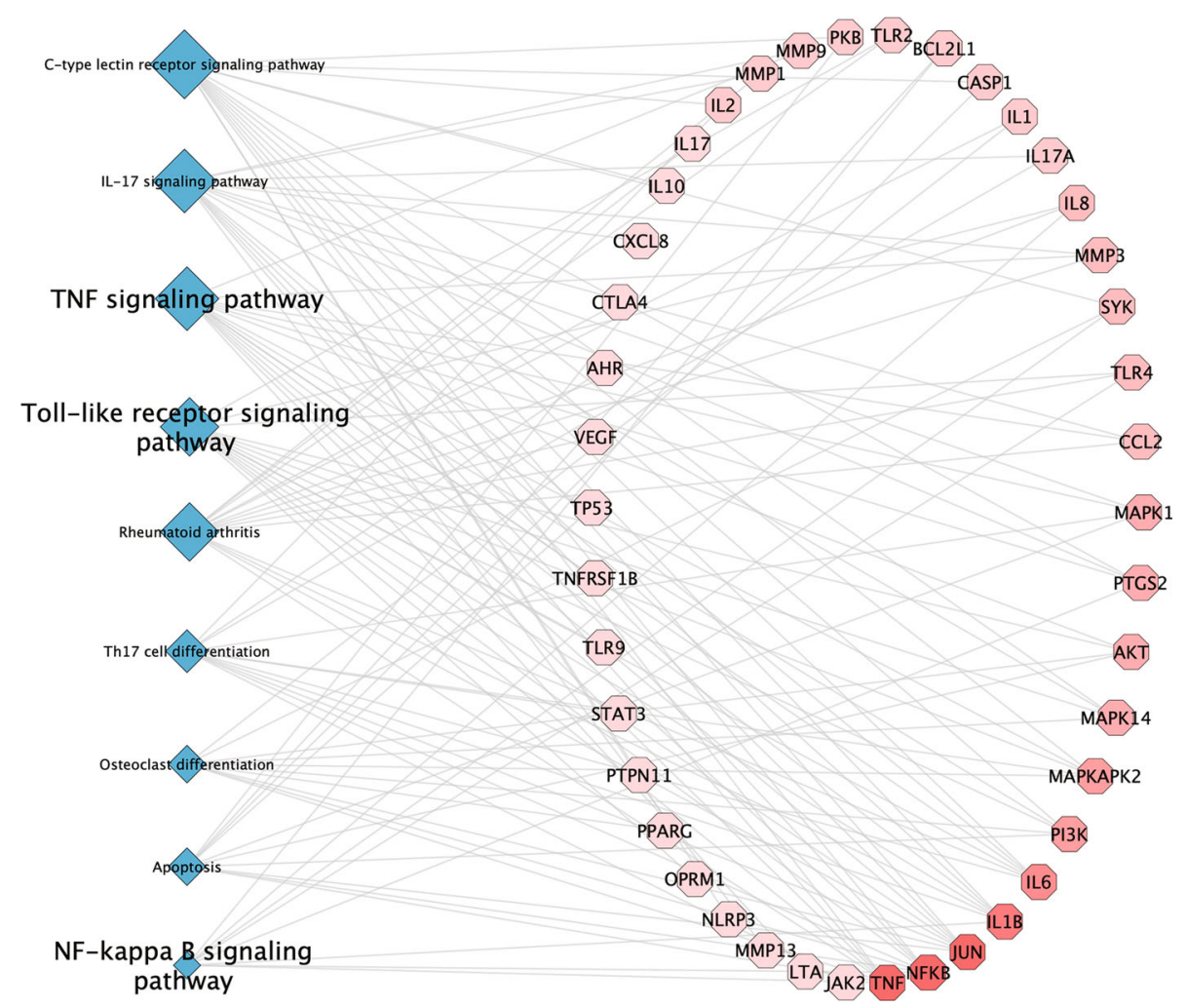

(d)

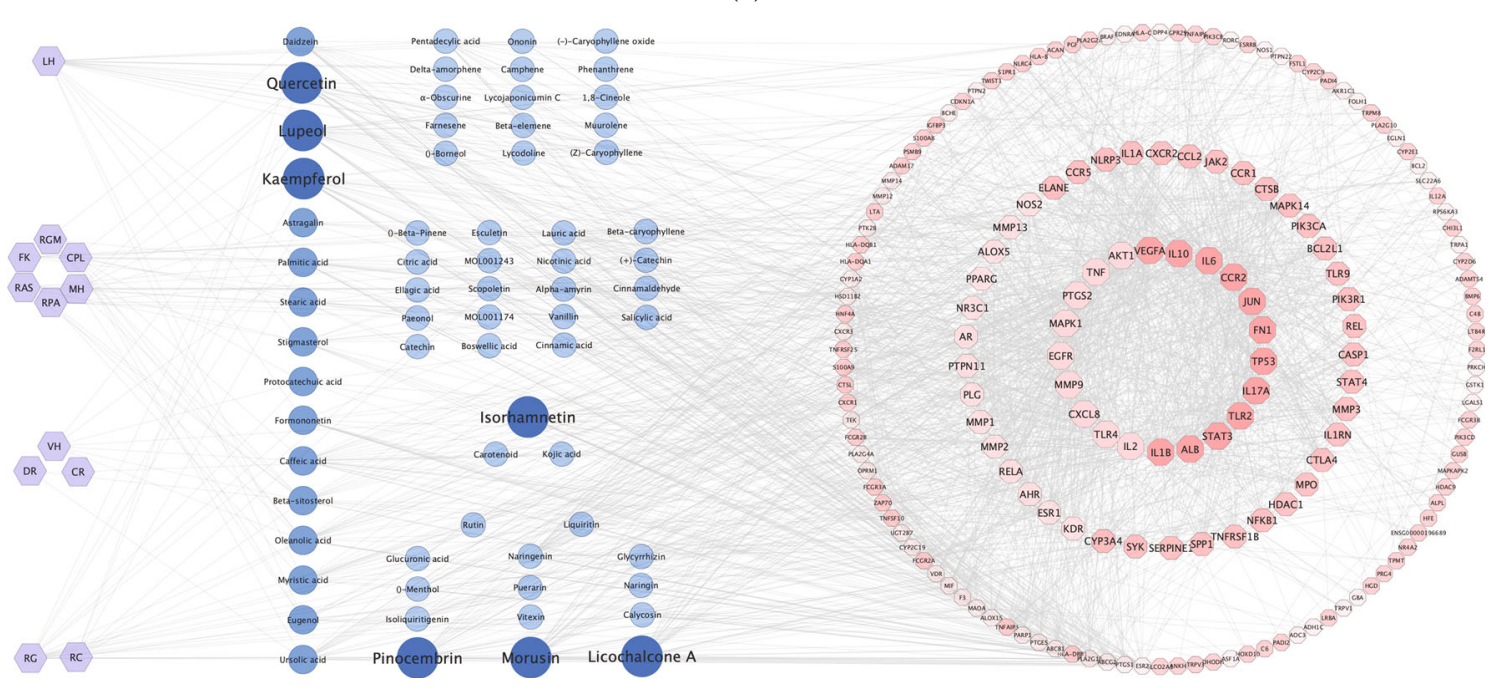

(e)

FIGURE 2: Network pharmacology analysis of the SHM formula. (a) Distribution of active phytochemicals in SHM herbs against OA. (b) The shared targets between SHM potential targets and OA targets. (c) Network of herb-active phytochemical target for SHM against OA. (d) Network of 63 key targets based on central network evaluation. The size of nodes is proportional to the degree centrality by topology analysis. (e) The top ten pathways identified by KEGG enrichment analysis and the corresponding key targets. SHM: shenjinhuoxue mixture; OA: osteoarthritis; KEGG: Kyoto Encyclopedia of Genes and Genomes Pathway database.

significantly inhibit TLR-4, IRAK1, and RANKL expression $(P<0.05, P<0.05$, and $P<0.01$, respectively), whereas middose SHM significantly reduced the expression of TLR- 4 and RANK (both $P<0.05$ ). Additionally, the expression of MMP3 was declined, but nonsignificantly in the SHM and PLQ groups (high vs. $\mathrm{C}_{\mathrm{OA}}, P=0.26$; $\mathrm{P}$ vs. $\mathrm{C}_{\mathrm{OA}}, P=0.29$ ).
(2) Amelioration of Cartilage Injury with the Reduced Expression of NF- $\kappa B-p 65$ and TRPV1 in SHM-Treated OA Rats

Immunohistochemical staining for nuclear NF- $\kappa \mathrm{B}-\mathrm{p} 65$ and TRPV1 for detecting their levels and distributions was performed in the synovial and cartilage tissue (Figure 5(c)). NF- $\kappa \mathrm{B}-\mathrm{p} 65$ and TRPV1 levels were 







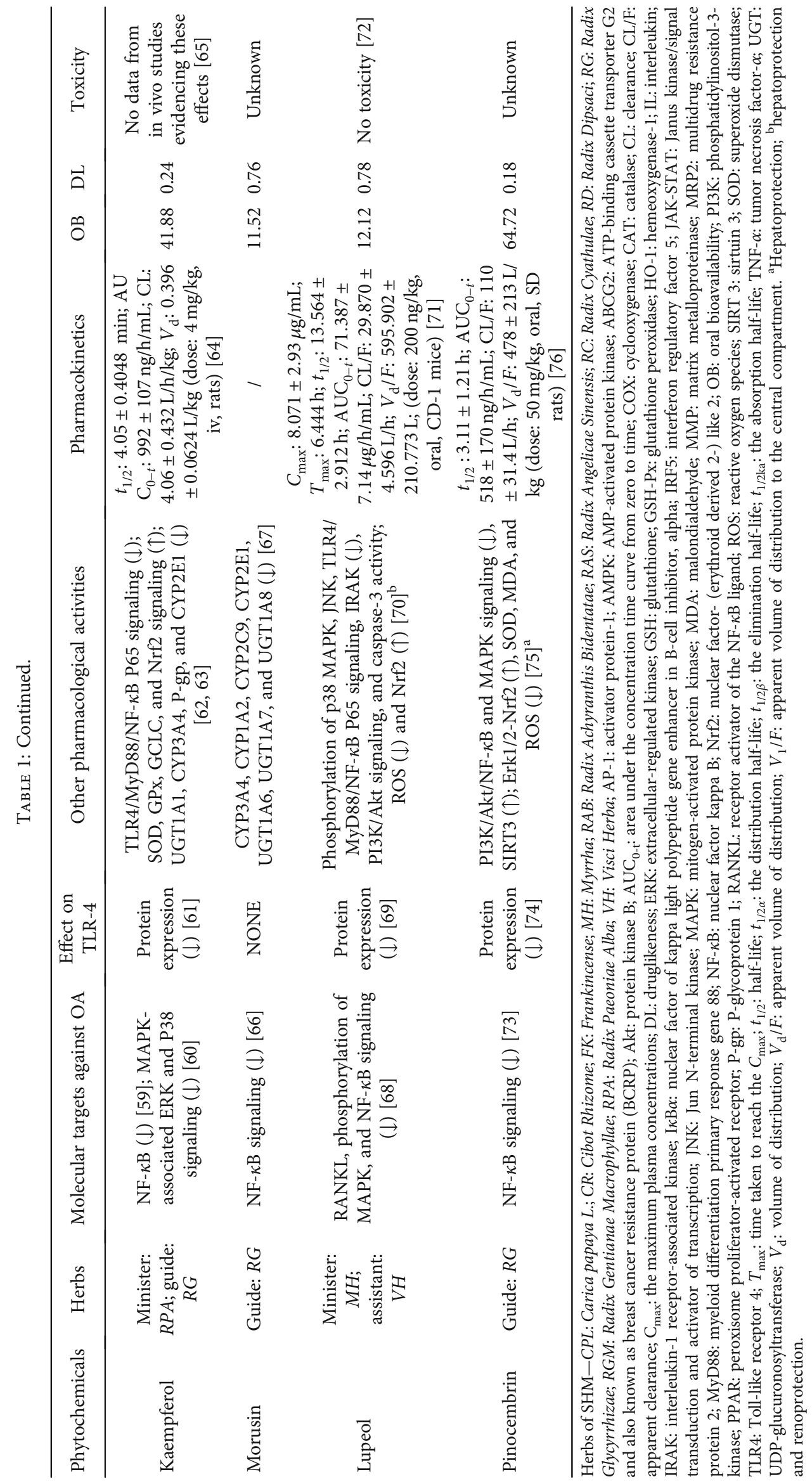



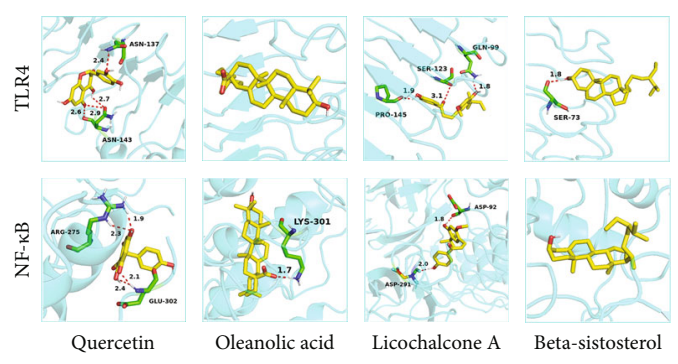

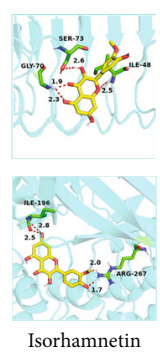

(a)
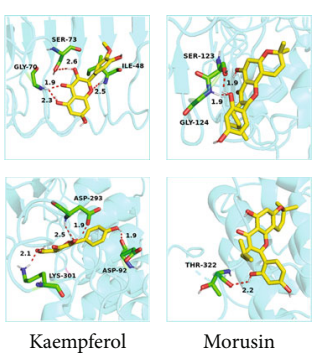

Morusin
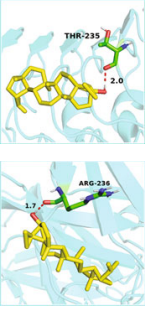

Lupeol

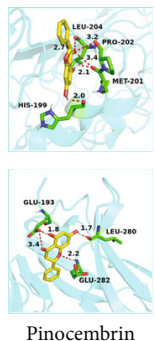

Pinocembrin

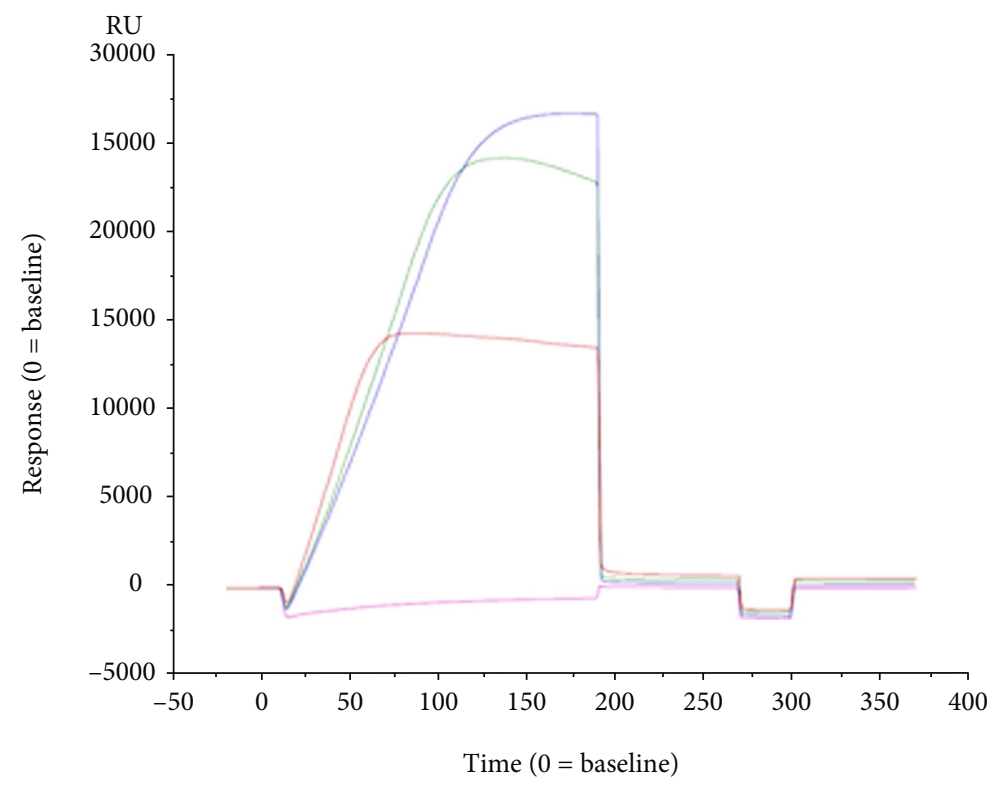

- $10 \mathrm{~mm}$ acetate 4

— $10 \mathrm{~mm}$ acetate 4.5

- $10 \mathrm{~mm}$ acetate 5

— $10 \mathrm{~mm}$ acetate 5.5

(b)
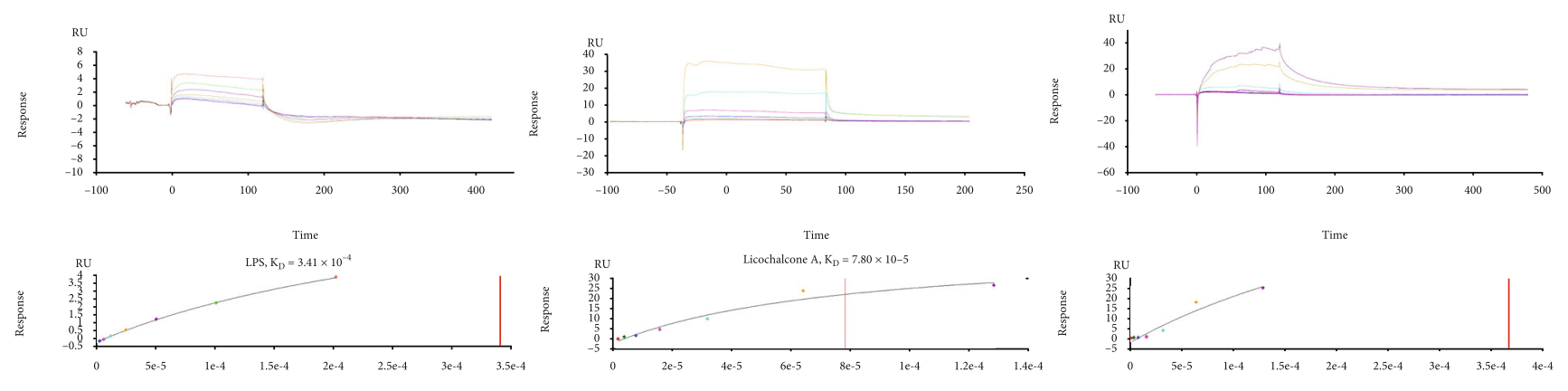

(c)

FIGURE 3: Interactions between the key active phytochemicals and the pivotal targets of TLR-4 and NF- $\kappa$ B. (a) Molecular models of the nine key active phytochemicals (molecule ligands) binding to the proteins of NF- $\kappa \mathrm{B}$ and TLR-4. The key active phytochemicals are shown interacting with the 3D structures of proteins, represented by the yellow stick models. Green and blue lines represent residues in the binding sites. The red dashed lines demarcate hydrogen bonds, and the interaction distances are indicated next to the bonds. (b) Sodium acetate pH 5.0 was the optimal condition to dilution TLR-4 with the highest bond response of 3324.5 RU. (c) Affinity-sensing diagrams (on the top) and fitting curves (on the bottom) of a series of concentrations of LPS (positive reference standard), licochalcone A, and oleanolic acid compounds with TLR-4. NF- $\kappa$ B: nuclear factor kappa B; TLR-4: Toll-like receptor 4; LPS: lipopolysaccharide; KD: equilibrium dissociation constant; RU: response units. 
TABLE 2: Molecular docking scores and bonds of the key active phytochemicals against TLR-4 and NF- $\kappa$ B.

\begin{tabular}{|c|c|c|c|c|}
\hline \multirow[b]{2}{*}{ Phytochemicals } & \multicolumn{2}{|r|}{ TLR-4 } & \multicolumn{2}{|r|}{$\mathrm{NF}-\kappa \mathrm{B}$} \\
\hline & $\begin{array}{c}\text { Docking score } \\
(\mathrm{kcal} / \mathrm{Mol})\end{array}$ & Protein residues of hydrogen bond & $\begin{array}{l}\text { Docking score } \\
(\mathrm{kcal} / \mathrm{Mol})\end{array}$ & Protein residues of hydrogen bond \\
\hline Licochalcone A & -3.89 & PRO-145, SER-123, GLN-99 & -5.5 & ASP-92, ASP-291 \\
\hline Quercetin & -5.28 & ASN-137, ASN-143 & -4.86 & GLU-302, RG-275 \\
\hline Isorhamnetin & -5.65 & ILE-48, SER-273, GLY-70 & -3.86 & ILE-196, ARG-267 \\
\hline Kaempferol & -5.67 & SER-240, LYS-239 & -5.66 & LYS-301, ASP-293, ASP-92 \\
\hline Morusin & -6.63 & GLY-124, SER-123 & -4.76 & THR-322 \\
\hline Lupeol & -7.03 & THR-235 & -8.19 & ARG-263 \\
\hline Pinocembrin & -5.84 & LUE-204, PRO-202, MET-201, HIS-199 & -5.79 & GLU-193, LEU-280, GLU-282 \\
\hline Beta-sitosterol & -5.74 & SER-73 & -5.89 & 1 \\
\hline Oleanolic acid & -5.28 & 1 & -8.52 & LYS-301 \\
\hline
\end{tabular}

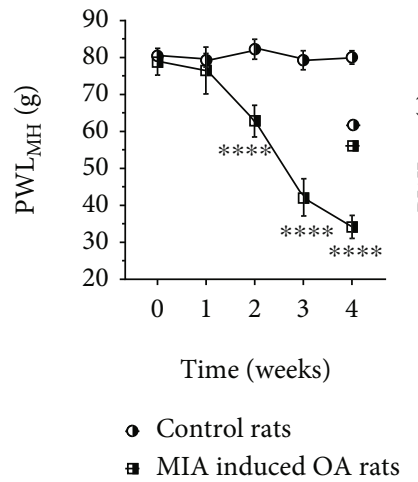

(a)

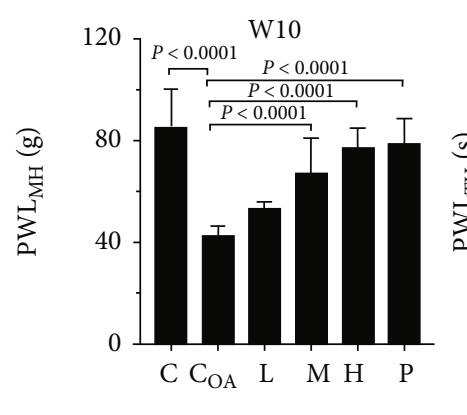

SHM

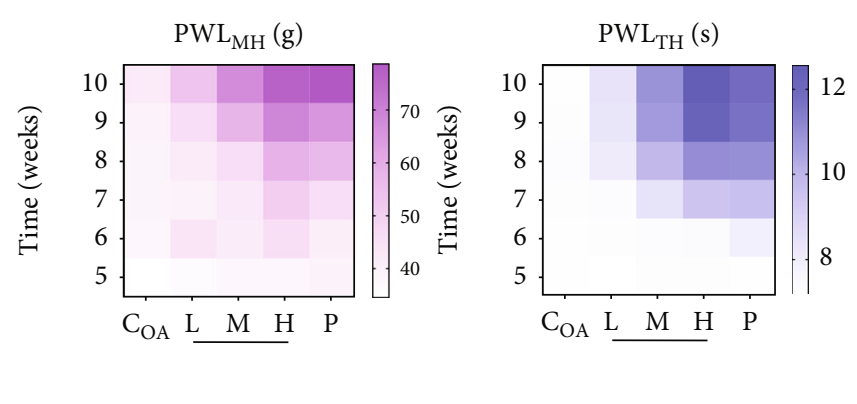

(b)

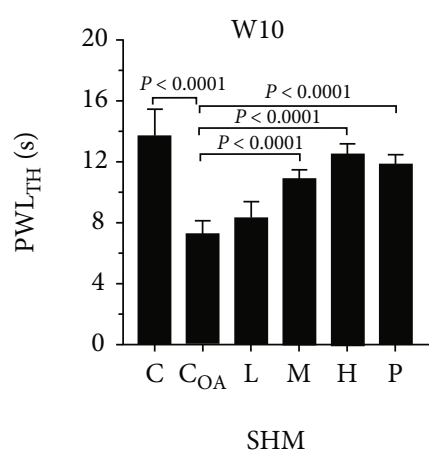

(c)

Figure 4: Analysis of mechanical hyperalgesia (MH) and thermal hyperalgesia (TH) in osteoarthritis (OA) rats. (a) Significant declines $(P<0.05$, weeks 2; $P<0.0001$, weeks 4$)$ of both paw withdrawal threshold ${ }_{\mathrm{MH}}\left(\mathrm{PWL}_{\mathrm{MH}}\right)$ and $\mathrm{PWL}_{\mathrm{TH}}$ in $\mathrm{OA}$ rats, compared to control rats, indicated the successful induction of OA by monosodium iodoacetate (MIA). (b) Heat maps of $\mathrm{PWL}_{\mathrm{MH}}$ and $\mathrm{PWL}_{\mathrm{TH}}$ in $\mathrm{OA}$ rats that received oral shenjinhuoxue mixture (SHM) showed that $\mathrm{MH}$ and $\mathrm{TH}$ were gradually improved as the dose and treatment course of SHM increased. (c) SHM interventions of mid and high doses significantly increased $(P<0.0001) \mathrm{PWL}_{\mathrm{MH}}$ and $\mathrm{PWL}_{\mathrm{TH}}$ in $\mathrm{OA}_{\text {rats }}$ at the end of pharmacology experiment (weeks 10).

significantly lower in all OA rats treated with high and mid doses of SHM (all $P<0.0001$; Figure 5(d)). The areas of cartilage injury in different groups were observed through TB staining (Figure 5(e)). The results indicated that SHM ameliorated cartilage degeneration, especially in the midand high-dose groups. Finally, the H\&E staining results
(Figure 5(f)) showed that high and mid doses of SHM remarkably mitigated knee joint injury.

3.2.2. Safety of Long-Term SHM Use in Rats. (1) No Significant Changes in Clinical Observations, Body Weight, and Food Consumption in SHM-Treated Rats 

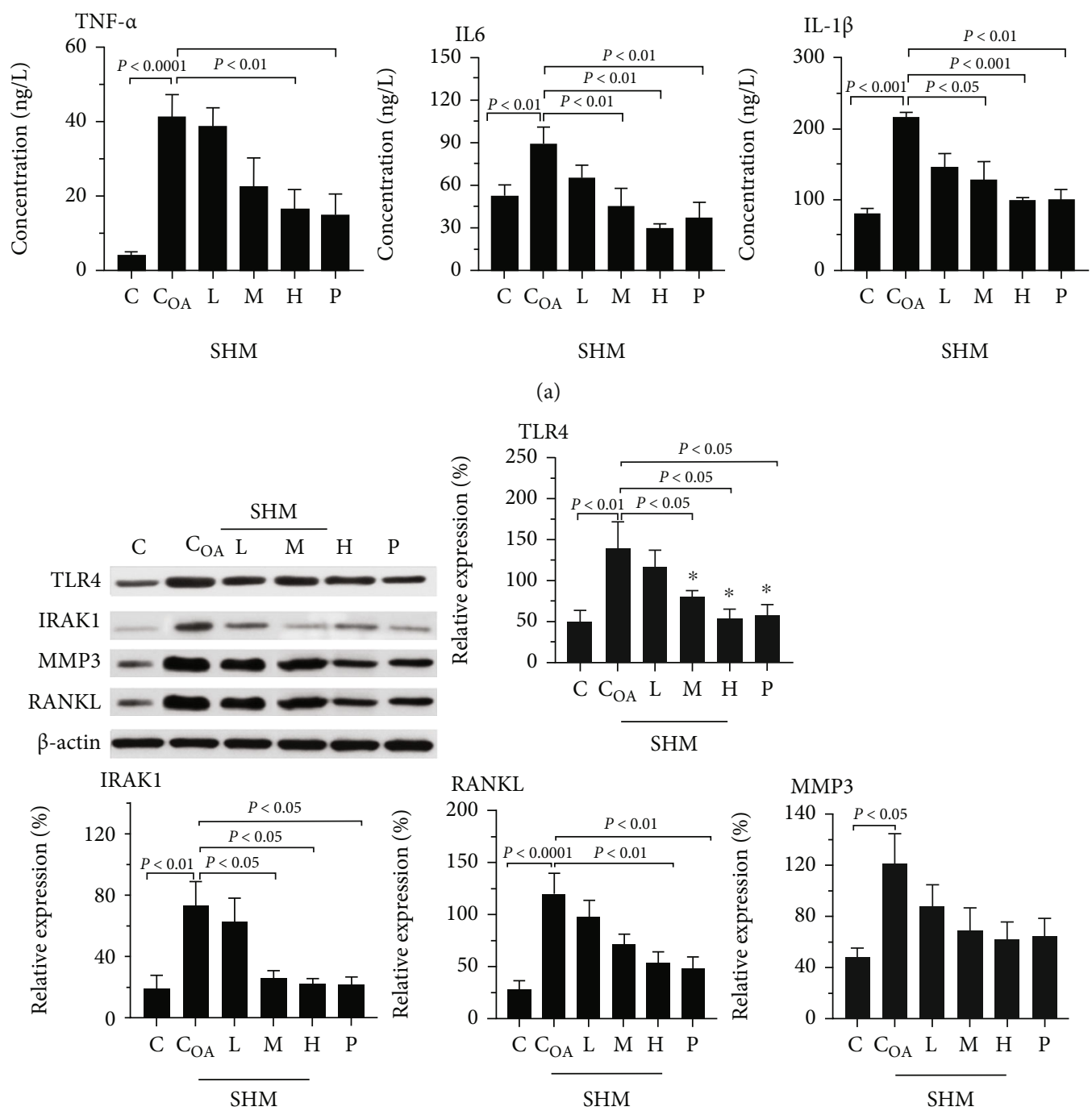

(b)

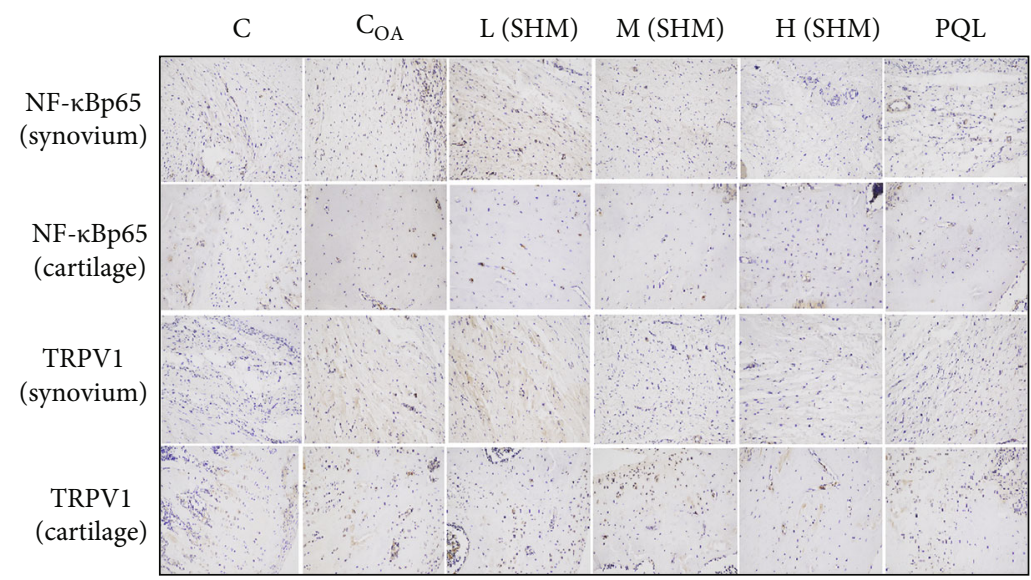

(c)

FIgURE 5: Continued. 

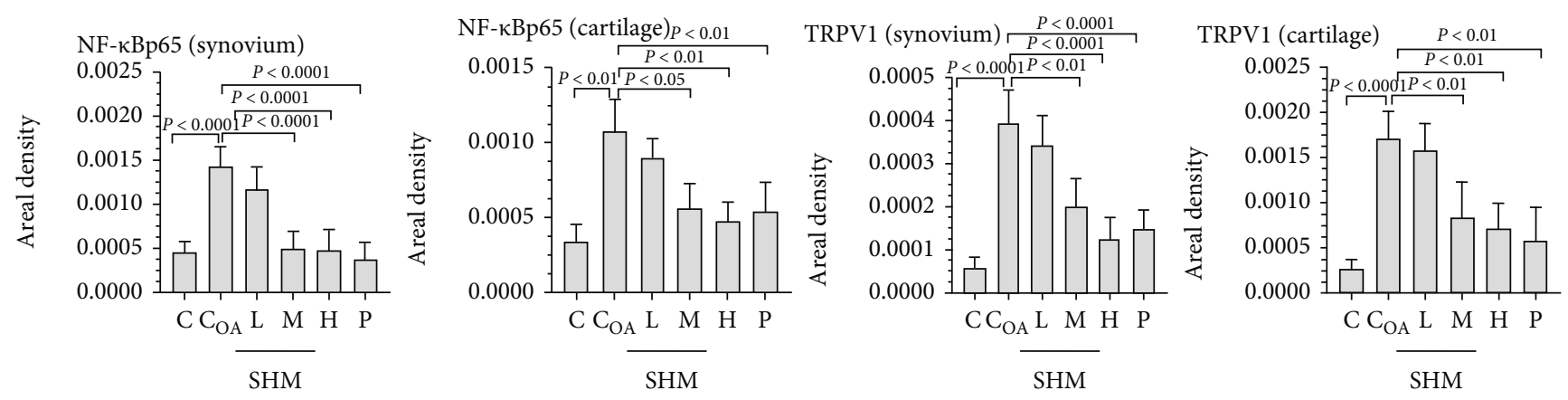

(d)

C

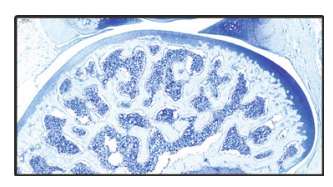

L (SHM)

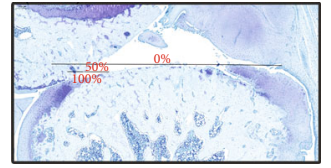

$\mathrm{C}_{\mathrm{OA}}$

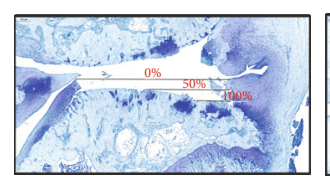

$\mathrm{M}$ (SHM)

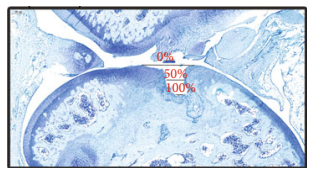

(e)

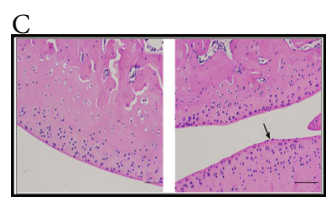

L (SHM)

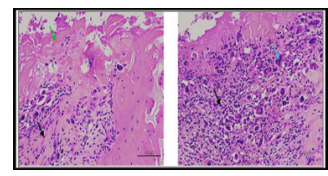

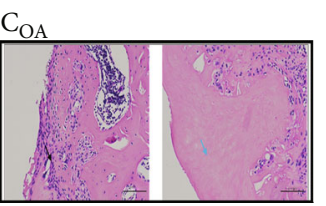

$\mathrm{M}(\mathrm{SHM})$

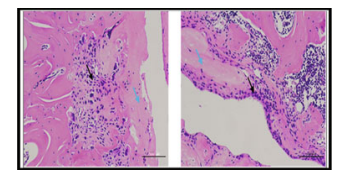

(f)
PLQ

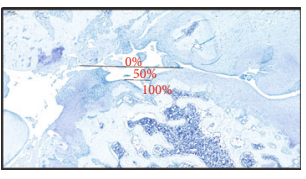

$\mathrm{H}$ (SHM)
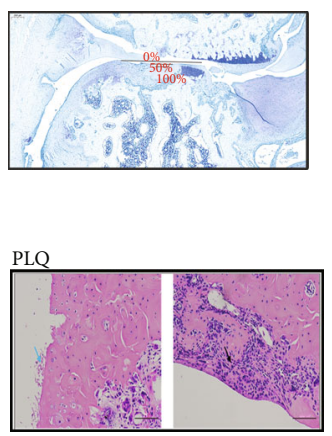

$\mathrm{H}$ (SHM)

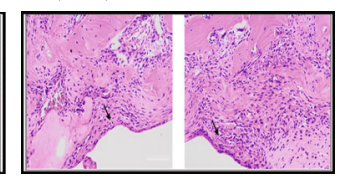

Figure 5: TLR4, RANKL, IRAK1, TNF- $\alpha$, IL-6, IL-1 $\beta$, and MMP3 levels of synovium and cartilage of OA rat knees in C, $\mathrm{C}_{\mathrm{OA}}$, SHM, and P groups. (a) TNF- $\alpha$, IL-6, and IL- $1 \beta$ levels were measured by ELISA kit according to the manufacturer's instructions. (b) TLR4, IRAK1, MMP3, and RANKL levels were measured and quantified by Western blot analysis with $\beta$-actin as a protein loading control. Pathological changes of synovial and cartilage of rat knees in pharmacology experiment. (c) Representative images of synovium and cartilage sections immunohistochemically stained for nuclear NF- $\kappa$ B-p65 and TRPV1 (dark brown) in OA rats. (d) Quantification of nuclear NF- $\kappa$ B-p65 and TRPV1 levels with their distributions was detected by immunohistochemically staining in synovium and cartilage $(n=3)$. Images of keen joints were stained by (e) TB and (f) H\&E.

Clinical signs of toxicity or mortality were not observed in the SHM and control groups during all 8 weeks. Body weight and body weight gain in both male and female rats in the SHM-treated groups were comparable with those of the control group rats $(P>0.05$, Figure $S 1 A)$.

Although the food consumption of male rats in the $\mathrm{H}_{\mathrm{T}}$ group decreased from W2 to W8, the mean weekly food consumption of male and female rats in the SHM-treated groups was generally comparable with that of rats in the control group $(P>0.05$, Figure S1B).

(2) No Abnormalities in Hematological and Biochemical Analyses of SHM Groups

Hematology of SHM-treated male and female rats was generally comparable with those of rats in the control group $(P>0.05)$. Although few values were significantly different between the groups, they were with their physiological range (Table S1). Similar results were observed for blood coagulation test and biochemical analysis (Table S2).

(3) Normal Organ Architectures in SHM-Treated Rats

Relative organ weights demonstrated nearly no differences between male and female rats administered with different SHM doses and $\mathrm{C}_{\mathrm{T}}$ groups on W8 and W11. Histopathological examinations of the heart, liver, spleen, lungs, and kidneys revealed normal architecture, indicating no morphologic disturbances in SHM-treated rats (Figure S1C).

\section{Discussion}

In our OA rats, $8.72 \mathrm{~g} / \mathrm{kg}$ and $26.2 \mathrm{~g} / \mathrm{kg}$ SHM orally significantly improved $\mathrm{MH}$ and $\mathrm{TH}(P<0.0001)$ and restored joint 
injury after 6 weeks of treatment. It relieved inflammation in the synovium and cartilage tissue, with the decreased levels of inflammatory mediators, including IRAK1, TNF- $\alpha$, IL-6, and IL- $1 \beta$, via the inhibition of TLR- 4 and NF- $\kappa$ B activation. Moreover, the declined expression of RANKL and TRPV1 reduced osteoarthritic osteoblast production and pain. We also identified the key active phytochemicals of SHM, including $\beta$-sitosterol, oleanolic acid, licochalcone A, quercetin, isorhamnetin, kaempferol, morusin, lupeol, and pinocembrin. Their interactions with TLR- 4 and NF- $\kappa$ B were also investigated. In particular, licochalcone A and oleanolic acid were verified to have TLR-4-binding affinity. In the chronic toxicity experiment, SHM at $60 \mathrm{mg} / \mathrm{kg}$ remained safe in our rats even after 2 months of intervention.

SHM exerted synergistic anti-inflammatory activity in the treatment of OA through multiple active phytochemicals with multitarget effects on the inhibition of the TLR-4 and NF- $\kappa$ B pathways and the PK behaviors. TLR- 4 is the pivotal receptor regulating $\mathrm{OA}$ inflammation onset and progress via NF- $\kappa$ B pathway activation [77]. Next, the activated NF- $\kappa$ B is the central contributory factor, accompanied by reactive oxygen species (ROS) generation, in joint inflammation and tissue destruction [78]. TLR-4 can also promote OA progression by activating M1 polarization of synovial macrophages with IL- $1 \beta$ and TNF- $\varnothing$ generation [79]. Notably, our SPR assay showed that licochalcone $\mathrm{A}$ and oleanolic acid directly inhibited TLR4 $\left(K_{\mathrm{D}}=7.80 \times 10^{-5}\right.$ and $\left.3.66 \times 10^{-4}\right)$, exhibiting the antagonistic effects. In line with this, licochalcone A can inhibit the TLR4/NF- $\kappa$ B pathway and efficiently alleviate inflammatory response in mice with LPS-induced acute liver injury [41]. Oleanolic acid can reduce the levels of TLR-4 and its downstream NF- $\kappa$ B in Salmonella typhimurium-induced mouse diarrhea [36]. In addition, lupeol shows good affinity with TLR4 in the molecular docking model. Lupeol downregulated the mRNA and protein expressions of TLR4 with the inhibition of the downstream MyD88 and NF- $\kappa \mathrm{B}$, restraining the release of IL- $1 \beta$ and TNF- $\bigotimes$, in viral myocarditis mice [69]. Isorhamnetin can block the LPS-TLR-4 interaction, alleviate inflammatory responses, and reduce ROS generation in BV2 microglia [55]. Moreover, the other active phytochemicals except morusin inhibited the expression of TLR-4 and elicited their anti-inflammatory activities (Table 1). Of these phytochemicals, quercetin can induce the alternatively activated type 2 (M2) polarization of synovial macrophages, inhibiting inflammation and apoptosis of chondrocytes for cartilage repair after OA [45]. Lu et al. [49] reported that quercetin inhibited the TLR4/MyD88/NF- $\kappa \mathrm{B}$ signaling pathway, caused the inhibition of M1 macrophage polarization, and exerted renoprotective effects. Thus, these natural inhibitors and/or potential antagonists of TLR-4 may modulate NF- $\kappa$ B activation and M1/M2 polarizations of macrophages, favoring the use of SHM to treat OA. Most key active phytochemicals were multitarget inhibitors of the NF- $\kappa$ B pathway and exhibited synergistic anti-OA properties. These SHM phytochemicals could suppress NF- $\kappa \mathrm{B}$ activation in combination with Nrf2, MAPK, and RANKL, alleviating ROS generation, chondrocyte catabolism, and osteoclast dif- ferentiation, respectively. Moreover, Yang et al. [80] recently identified that lycopodium alkaloids, such as lycojaponicumin $\mathrm{C}$, from the principal of $L H$ have anti-inflammatory effects, which are positively correlated with the chromatographic peak, through AChE/NF- $\kappa \mathrm{B}$ pathway suppression in rats with rheumatoid arthritis. However, the effect of lycopodium alkaloids against OA remains insufficient and warrants further research. The anti-inflammatory activity of SHM also depends on the PK behaviors of active phytochemicals. Lycopodium alkaloids show rapid absorption with a $T_{\max }$ of $0.79-1.58 \mathrm{~h}$ after intragastric administration of $\mathrm{LH}$ extract in rats [28]. Oleanolic acid, quercetin, isorhamnetin, and lupeol are slowly absorbed into the blood with a long $T_{\max }$ of 3-7.2 h. Kaempferol has high clearance with $t_{1 / 2}$ of $4.05 \pm 0.4048 \mathrm{~min}$ and CL of $4.06 \pm 0.432 \mathrm{~L} / \mathrm{h}$ $/ \mathrm{kg}$ [64]. However, the other phytochemicals are long-acting active phytochemicals with low clearance (e.g., lupeol with $t_{1 / 2}=13.564 \pm 2.912 \mathrm{~h}$ and $\left.\mathrm{CL} / \mathrm{F}=29.870 \pm 4.596 \mathrm{~L} / \mathrm{h}\right)[71]$. SHM might provide the ongoing anti-inflammatory concentration of active phytochemical for OA therapy, attributing to their difference in PK profiles. Additionally, oleanolic acid, lupeol, and pinocembrin, with high $V_{\mathrm{d}}$ values of $3371.1 \pm 1990.1 \mathrm{~L}$, $595.902 \pm 210.773 \mathrm{~L}$, and $478 \pm 213 \mathrm{~L} / \mathrm{kg}$ might be distributed widely, indicating high concentrations in OA rat knees.

Furthermore, active phytochemicals of SHM have altered PK profiles after interactions with gut microbiota, membrane transporters, and metabolizing enzymes and these altered phytochemicals synergistically exert antiinflammatory activity against OA. The active phytochemicals of SHM with low OB were commonly not absorbed well and interacted with gut microbiota. Although ursolic acid has a poor $\mathrm{OB}(16.77 \%)$, it showed high pharmacological actions, related to its active metabolites transformed by intestinal microbes [81]. Arnoriaga-Rodríguez et al. [82] reported that lupeol $(\mathrm{OB}=12.12 \%)$ positively regulated the gut bacterial ecosystem via a ClpB-like gene function. Accordingly, inflammation in OA is alleviated through the systemic action of gut microbiota regulated by the phytochemicals [83]. Moreover, the anti-inflammatory activity of SHM may be increased via the effects of active phytochemicals on CYP3A4 and P-gp, which are involved in the modulation of first-pass metabolism and enhancement of oral absorption. CYP3A4 and P-gp are located on the apical membrane of the small intestinal cells [84]. CYP3A4 is a key target in the assistant and guide functions of SHM against OA. It is the most abundant hepatic and intestinal phase I enzyme, which catalyzes the metabolism of a wide variety of endogenous and exogenous compounds [85]. We noted that the enzyme activity of CYP3A4 was inhibited by the key active phytochemicals of SHM. However, glycyrrhizin, the main active phytochemical in $R G$ (proportion $=19 \%$ ), can increase CYP3A4 activity by approximately $30 \%$ [86]. Some of the key active phytochemicals, especially those in $R G$, can lower the activity of P-gp, potentially inhibiting efflux transport of potential substrate phytochemicals [87]. P-gp is also expressed in the synovial tissue and can influence the joint disposition of drugs [88]. We also found the regulatory effects of the active SHM phytochemicals on CYP1A, CYP2C, UGT1A, 


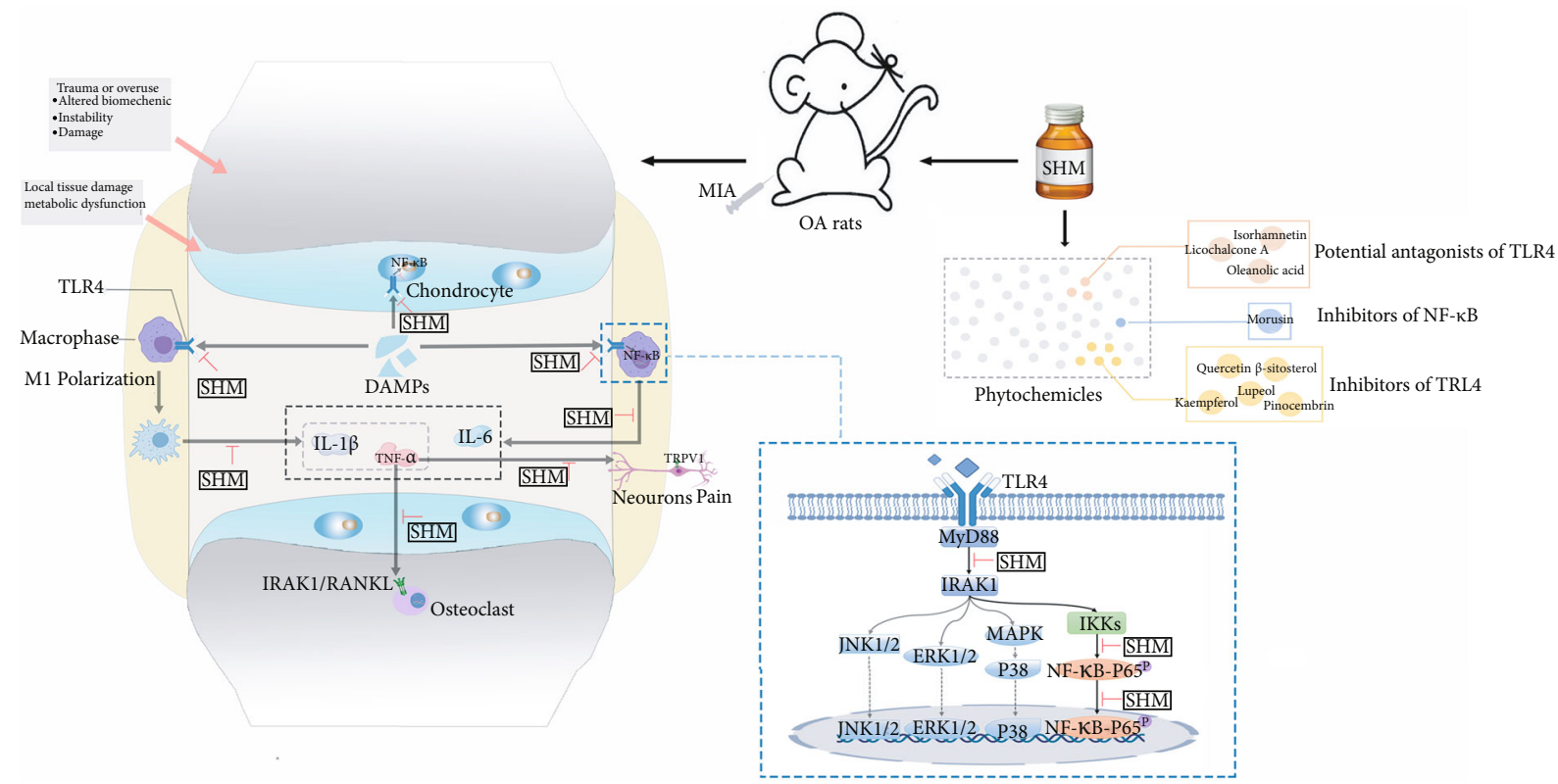

FIGURE 6: Mechanism underlying treatment of SJHX mixture against OA via inhibiting TLR4 and NF- $\kappa \mathrm{B}$ activation.

BCRP, MRP2, and so on. Interplays between the active GRR phytochemicals and other ingredients via CYP450s and membrane transporters in vivo may uncover the role of the guide on transportation of drug to the desired site in SHM.

Long-term use of SHM is safe even at a high dose. Moreover, SHM has a detoxifying function of acetaminophen (APAP) that is an over-the-counter (OTC) analgesic and recommended by all guidelines as the first line of treatment for people with mild to moderate OA [89]. High-dose SHM at $60 \mathrm{~g} / \mathrm{kg}$, based on the effective doses of 8.72 and $26.2 \mathrm{~g} / \mathrm{kg}$ (in the $\mathrm{M}$ and $\mathrm{H}$ groups, respectively) remained nontoxic even after 8 weeks of intragastric administration in rats. The key active phytochemicals of SHM itself had no toxicity ( $\beta$-sitosterol, isorhamnetin, kaempferol, and lupeol) [27, 58, 72 ] or has low toxicity (oleanolic acid, LD50 = $90 \mathrm{mg} / \mathrm{kg}$; licochalcone A, $9 \mu \mathrm{g} / \mathrm{mL}$ (MTT cell viability assays); quercetin, $>1500 \mathrm{mg} /$ day with nephrotoxicity). Safety of SHM can somewhat be attributed to its complementary combination of active phytochemicals and targets against OA. The key active phytochemicals of SHM can prevent APAP-induced acute hepatotoxicity or nephrotoxicity by inhibiting the $\mathrm{NF}-\kappa \mathrm{B}$ and PI3K/Akt pathways or by activating Nrf2 [89-93]. Through the suppression of NF- $\kappa \mathrm{B}$ and PI3K/Akt pathways, the antiapoptotic activity of the key active phytochemicals can alleviate hepatic necrosis induced by APAP [90]. Because the activation of the PI3K/Akt pathway enhances the transcriptional activity of NF- $\kappa \mathrm{B}$ via the activation of $\mathrm{I} \kappa \mathrm{B}$ kinase $(\mathrm{IKK} \alpha / \beta)$ [91]. NF- $\kappa \mathrm{B}$ not only participates in the process of immunity and inflammation but also causes apoptosis by regulating apoptotic genes [92]. Furthermore, because of the interactions of ROS with $\mathrm{NF}-\kappa \mathrm{B}$ and PI3K, the antioxidant activity of these active phytochemicals via the activation of the $\mathrm{Nrf} 2$ pathway can relieve inflammation and apoptosis $[26,32,38,50,56,62,72,75,88]$. The pleiotropic activities of the active phytochemicals indicate thus the safety of SHM when it is used alone or in combination with APAP.

This study is limited by the effect of SHM on the expression of osteoprotegerin (OPG), with regard to clarifying OPG's role in the osteochondral crosstalk of OA. As a decoy receptor that binds to RANKL, OPG provides the antiosteoclastogenesis activity. Additionally, the therapeutic effects of SHM against OA are closely related to its active phytochemical contents that may differ in different batches, attributing to the quality difference of herbs in SHM. Although we have identified the key active phytochemicals of SHM and verified their interaction with TLR- 4 and NF- $\kappa$ B. Further study is needed to identify the reliable quality markers of SHM from these active phytochemicals for the establishment of the scientific system of SHM quality evaluation [93]. Moreover, future research clarifying the synergistic mechanism of active phytochemicals with the precise dosage in SHM is also warranted.

\section{Conclusions}

In summary, our results demonstrated that SHM, derived from the empirical TCM prescription at our hospital, attenuated inflammation, pain, and cartilage degeneration via inhibiting TLR-4 and NF- $\kappa \mathrm{B}$ activation in OA rats (the underlying mechanism shown in Figure 6). Moreover, long-term SHM use is safe. The key active phytochemicals in SHM were found to be $\beta$-sitosterol, oleanolic acid, licochalcone A, quercetin, isorhamnetin, kaempferol, morusin, lupeol, and pinocembrin. Furthermore, licochalcone A and oleanolic acid have antagonistic effect on TLR-4, as verified through their binding affinity. Our study provides an experimental foundation for the development of SHM into a more effective dosage form or DMOADs. 


\section{Data Availability}

The data used to support the results of this study are included within the article.

\section{Conflicts of Interest}

The authors declare that they have no conflicts of interest.

\section{Authors' Contributions}

Jingjing Huang, Wanhua Yang, and Xiaofei Chen conceptualized and designed the study. Xiaoqin Ma and Chenxia Hao performed the study and acquired and analyzed the data. Xiaoqin Ma and Chenxia Hao contributed equally to this work. Zhaokang Zhang, Huiting Jiang, and Weixia Zhang interpreted the data. Xiaoqin Ma drafted the manuscript. Jingjing Huang and Wanhua Yang further wrote/revised the manuscript.

\section{Acknowledgments}

The authors thank Professor Longhai Shen and the staffs in Shanghai Institute of Pharmaceutical Industry for their valuable guidance and support in the research work. This study is sponsored by the key scientific research projects of the Science and Technology Commission of Shanghai (17401901100) and the Science and Technology Innovation Project of the Traditional Chinese Medicine of Shanghai Municipal Commission of Health (ZYKC201701007).

\section{Supplementary Materials}

(1) Chemicals and regents of surface plasmon resonance (SPR) assay, (2) preparation method of shenjinhuoxue mixture (SHM), and (3) calculation of low, mid, and high SHM dosages of rats was listed in the file of "Supplementary Materials." Table S1: hematologic analysis of male rats (A) and female rats (B) in the chronic toxicity experiment. Table S2: blood coagulation test and clinical biochemistry analysis of male rats (A) and female rats (B) in the chronic toxicity experiment. Figure S1: S1 results of chronic systemic toxicity experiment. (A) Body weight changes and (B) food consumption changes of male and female rats. (C) Histologic observations of the heart, liver, spleen, lung, and kidney in $\mathrm{C}_{\mathrm{T}}$ and $\mathrm{H}_{\mathrm{T}}$ groups on week 8 and week 11. (Supplementary Materials)

\section{References}

[1] A. Nelson, "Osteoarthritis year in review 2017: clinical," Osteoarthritis and cartilage., vol. 26, no. 3, pp. 319-325, 2018.

[2] B. Xia, J. Z. Di Chen, S. Hu, H. Jin, and P. Tong, "Osteoarthritis pathogenesis: a review of molecular mechanisms," Calcified Tissue International, vol. 95, no. 6, pp. 495-505, 2014.

[3] W. H. Robinson, C. M. Lepus, Q. Wang et al., "Low-grade inflammation as a key mediator of the pathogenesis of osteoarthritis," Nature Reviews Rheumatology, vol. 12, no. 10, pp. 580-592, 2016.

[4] J. Woodell-May and S. Sommerfeld, "Role of inflammation and the immune system in the progression of osteoarthritis,"
Journal of Orthopaedic Research: Official Publication of the Orthopaedic Research Society, vol. 38, no. 2, pp. 253-257, 2020.

[5] R. Gómez, A. Villalvilla, R. Largo, O. Gualillo, and G. HerreroBeaumont, "TLR4 signalling in osteoarthritis-finding targets for candidate DMOADs," Nature reviews Rheumatology, vol. 11, no. 3, pp. 159-170, 2015.

[6] P. Burrage, K. Mix, and C. Brinckerhoff, "Matrix metalloproteinases: role in arthritis," Frontiers in Bioscience: A Journal and Virtual Library, vol. 11, no. 1, pp. 529-543, 2006.

[7] Y. Zhang and O. Igwe, "Exogenous oxidants activate nuclear factor kappa B through Toll-like receptor 4 stimulation to maintain inflammatory phenotype in macrophage," Biochemical Pharmacology, vol. 147, pp. 104-118, 2018.

[8] A. Marahleh, H. Kitaura, F. Ohori et al., "TNF- $\alpha$ directly enhances osteocyte RANKL expression and promotes osteoclast formation," Frontiers in Immunology, vol. 10, 2019.

[9] X. Zhou, H. Cao, Y. Yuan, and W. Wu, "Biochemical signals mediate the crosstalk between cartilage and bone in osteoarthritis," BioMed Research International, vol. 2020, Article ID 5720360, 8 pages, 2020.

[10] H. Schaible, "Nociceptive neurons detect cytokines in arthritis," Arthritis Research \& Therapy., vol. 16, no. 5, p. 470, 2014.

[11] Y. Wang, Y. Gao, and Q. Tian, "Author Correction: TRPV1 SUMOylation regulates nociceptive signaling in models of inflammatory pain," Nature Communications, vol. 9, no. 1, 2018.

[12] W. Oo, S. Yu, M. Daniel, and D. Hunter, "Disease-modifying drugs in osteoarthritis: current understanding and future therapeutics," Expert Opinion on Emerging Drugs, vol. 23, no. 4, pp. 331-347, 2018.

[13] Y. Henrotin and A. Mobasheri, "Natural products for promoting joint health and managing osteoarthritis," Current Rheumatology Reports, vol. 20, no. 11, 2018.

[14] D. Dragos, M. Gilca, L. Gaman et al., "Phytomedicine in joint disorders," Nutrients, vol. 9, no. 1, p. 70, 2017.

[15] S. Ansaripour and M. Dehghan, "Efficacy of some herbal medicines in osteoarthritis with a focus on topical agents: a systematic review," Current Pharmaceutical Design, vol. 26, no. 22, pp. 2676-2681, 2020.

[16] Z. Zhang, C. Huang, Q. Jiang et al., "Guidelines for the diagnosis and treatment of osteoarthritis in China (2019 edition)," Annals of Translational Medicine, vol. 8, no. 19, p. 1213, 2020.

[17] N.-D. Zhang, T. Han, B.-K. Huang et al., "Traditional Chinese medicine formulas for the treatment of osteoporosis: implication for antiosteoporotic drug discovery," Journal of Ethnopharmacology, vol. 189, pp. 61-80, 2016.

[18] L. Wang, X.-F. Zhang, X. Zhang et al., "Evaluation of the therapeutic effect of traditional chinese medicine on osteoarthritis: a systematic review and meta-analysis," Pain Research and Management, vol. 2020, Article ID 5712187, 23 pages, 2020.

[19] M. Wang, L. Liu, C. S. Zhang et al., "Mechanism of traditional chinese medicine in treating knee osteoarthritis," Journal of Pain Research, vol. Volume 13, pp. 1421-1429, 2020.

[20] X. Luan, L.-J. Zhang, X.-Q. Li et al., "Compound-based Chinese medicine formula: from discovery to compatibility mechanism," Journal of Ethnopharmacology, vol. 254, p. 112687, 2020.

[21] M.-X. Yu, X.-Q. Ma, X. Song et al., "Validation of the key active ingredients and anti-inflammatory and analgesic effects of shenjin huoxue mixture against osteoarthritis by integrating network pharmacology approach and thin-layer chromatography 
analysis," Drug Design, Development and Therapy, vol. Volume 14, pp. 1145-1156, 2020.

[22] A. Franceschini, D. Szklarczyk, and S. Frankild, "STRING v9.1: protein-protein interaction networks, with increased coverage and integration," Nucleic Acids Research, vol. 41, 2012.

[23] S. Rizvi, S. Shakil, and M. Haneef, "A simple click by click protocol to perform docking: AutoDock 4.2 made easy for nonbioinformaticians," EXCLI Journal, vol. 12, 2013.

[24] R. Sparks, J. Jenkins, and R. Fratti, "Use of surface plasmon resonance (SPR) to determine binding affinities and kinetic parameters between components important in fusion machinery," Methods in Molecular Biology (Clifton, N.J.), vol. 1860, 2019.

[25] G. W. Stevenson, H. Mercer, J. Cormier et al., "Monosodium iodoacetate-induced osteoarthritis produces pain-depressed wheel running in rats: implications for preclinical behavioral assessment of chronic pain," Pharmacology, Biochemistry, and Behavior, vol. 98, no. 1, pp. 35-42, 2011.

[26] R.-Z. Liu, C.-X. Fan, Z.-L. Zhang et al., "Effects of Dl-3-nbutylphthalide on cerebral ischemia infarction in rat model by mass spectrometry imaging," Int J Mol Sci, vol. 18, no. 11, p. 2451, 2017.

[27] B. Snider, A. Geiser, X.-p. Yu et al., "Long-acting and selective oxytocin peptide analogs show antidiabetic and Antiobesity effects in male mice," Journal of the Endocrine Society, vol. 3, no. 7, pp. 1423-1444, 2019.

[28] D. Ma, X. Gu, X. Wang, Y. Liu, and X. Di, "Pharmacokinetic studies of three alkaloids in rats after intragastrical administration of Lycopodii Herba extract by LC-MS/MS," Molecules (Basel, Switzerland), vol. 24, no. 10, p. 1930, 2019.

[29] B. C. Y. Cheng, X.-Q. Fu, H. Guo et al., "The genus_Rosa_and arthritis: Overview on pharmacological perspectives," Pharmacological Research, vol. 114, pp. 219-234, 2016.

[30] M. Valerio, H. Minderman, T. Mace, and A. Awad, “ $\beta$-Sitosterol modulates TLR4 receptor expression and intracellular MyD88-dependent pathway activation in J774A.1 murine macrophages," Cellular Immunology, vol. 285, no. 1-2, pp. 76-83, 2013.

[31] Y. Yin, X. Liu, J. Liu et al., "Beta-sitosterol and its derivatives repress lipopolysaccharide/d-galactosamine-induced acute hepatic injury by inhibiting the oxidation and inflammation in mice," Bioorganic \& Medicinal Chemistry Letters, vol. 28, no. 9, pp. 1525-1533, 2018.

[32] M. M. Leitao, J. A. S. Radai, I. C. Ferrari et al., "Effects of an ethanolic extract and fractions from _Piper glabratum_ (Piperaceae) leaves on pain and inflammation," Regulatory Toxicology and Pharmacology: RTP, vol. 117, p. 104762, 2020.

[33] J. Bao, W. Yan, K. Xu et al., "Oleanolic Acid Decreases IL- $1 \beta$ Induced Activation of Fibroblast-Like Synoviocytes via the SIRT3-NF- $\kappa \mathrm{B}$ Axis in Osteoarthritis," Oxidative Medicine and Cellular Longevity, vol. 2020, 10 pages, 2020.

[34] X. Kang, Z. Yang, J. Sheng et al., "Oleanolic acid prevents cartilage degeneration in diabetic mice via PPAR $\gamma$ associated mitochondrial stabilization," Biochemical and Biophysical Research Communications, vol. 490, no. 3, pp. 834-840, 2017.

[35] D. Zhao, B. Shu, C. Wang et al., "Oleanolic acid exerts inhibitory effects on the late stage of osteoclastogenesis and prevents bone loss in osteoprotegerin knockout mice," Journal of Cellular Biochemistry, vol. 121, no. 1, pp. 152-164, 2020.

[36] N. Dong, C. Xue, L. Zhang et al., "Oleanolic acid enhances tight junctions and ameliorates inflammation in salmonella typhimurium-induced diarrhea in mice via the TLR4/NF- $\kappa \mathrm{B}$ and MAPK pathway," Food \& function., vol. 11, no. 1, pp. 1122-1132, 2020.

[37] J. Liu, Y.-F. Lu, Q. Wu, S.-F. Xu, F.-G. Shi, and C. D. Klaassen, "Oleanolic acid reprograms the liver to protect against hepatotoxicants, but is hepatotoxic at high doses," Liver International: Official Journal of the International Association for the Study of the Liver, vol. 39, no. 3, pp. 427-439, 2019.

[38] M. Song, T.-j. Hang, Y. Wang et al., "Determination of oleanolic acid in human plasma and study of its pharmacokinetics in Chinese healthy male volunteers by HPLC tandem mass spectrometry," Journal of Pharmaceutical and Biomedical Analysis, vol. 40, no. 1, pp. 190-196, 2006.

[39] J. Liu, Y.-F. Lu, Y. Zhang, K. C. Wu, F. Fan, and C. D. Klaassen, "Oleanolic acid alters bile acid metabolism and produces cholestatic liver injury in mice," Toxicology and Applied Pharmacology, vol. 272, no. 3, pp. 816-824, 2013.

[40] S. Kim, M. Kim, Y. Min, and S. Kim, "Licochalcone A inhibits the formation and bone resorptive activity of osteoclasts," Cell Biology International, vol. 32, no. 9, pp. 1064-1072, 2008.

[41] H. Lv, H. Yang, Z. Wang et al., "Nrf2 signaling and autophagy are complementary in protecting lipopolysaccharide/d-galactosamine-induced acute liver injury by licochalcone A," Cell Death \& Disease, vol. 10, no. 4, p. 313, 2019.

[42] C.-J. Liou, Y.-K. Lee, N.-C. Ting et al., "Protective effects of licochalcone A ameliorates obesity and non-alcoholic fatty liver disease via promotion of the Sirt-1/AMPK pathway in mice fed a high-fat diet," Cells, vol. 8, no. 5, p. 447, 2019.

[43] X. Li, R. Sun, and R. Liu, "Natural products in licorice for the therapy of liver diseases: progress and future opportunities," Pharmacological Research, vol. 144, pp. 210-226, 2019.

[44] H. Si, C. Xu, J. Zhang et al., "Licochalcone A: An effective and low-toxicity compound against_Toxoplasma gondii in vitro_ and _in vivo_," International Journal for Parasitology. Drugs and Drug Resistance, vol. 8, no. 2, pp. 238-245, 2018.

[45] Y. Hu, Z. Gui, Y. Zhou, L. Xia, K. Lin, and Y. Xu, "Quercetin alleviates rat osteoarthritis by inhibiting inflammation and apoptosis of chondrocytes, modulating synovial macrophages polarization to M2 macrophages," Free Radical Biology \& Medicine, vol. 145, pp. 146-160, 2019.

[46] K. Feng, Z. Chen, L. Pengcheng, S. Zhang, and X. Wang, "Quercetin attenuates oxidative stress-induced apoptosis via SIRT1/AMPK-mediated inhibition of ER stress in rat chondrocytes and prevents the progression of osteoarthritis in a rat model," Journal of Cellular Physiology, vol. 234, no. 10, pp. 18192-18205, 2019.

[47] J. Zhang, J. Yin, D. Zhao et al., "Therapeutic effect and mechanism of action of quercetin in a rat model of osteoarthritis," The Journal of International Medical Research, vol. 48, no. 3, article 030006051987346, 2020.

[48] Y. Ge, K. Feng, and X. Liu, "Quercetin inhibits macrophage polarization through the $\mathrm{p}-38 \alpha / \beta$ signalling pathway and regulates OPG/RANKL balance in a mouse skull model," Journal of Cellular and Molecular Medicine, vol. 24, no. 5, pp. 32033216, 2020.

[49] H. Lu, L. Wu, L. Liu et al., "Quercetin ameliorates kidney injury and fibrosis by modulating M1/M2 macrophage polarization," Biochemical Pharmacology, vol. 154, pp. 203-212, 2018.

[50] G. Carullo, A. R. Cappello, L. Frattaruolo, M. Badolato, B. Armentano, and F. Aiello, "Quercetin and derivatives: 
useful tools in inflammation and pain management," Future Medicinal Chemistry, vol. 9, no. 1, pp. 79-93, 2017.

[51] V. Mohos, E. Fliszár-Nyúl, O. Ungvári et al., "Inhibitory effects of quercetin and its main methyl, sulfate, and glucuronic acid conjugates on cytochrome P450 enzymes, and on OATP, BCRP and MRP2 transporters," Nutrients, vol. 12, no. 8, p. 2306, 2020.

[52] Y. Moon, L. Wang, R. DiCenzo, and M. Morris, "Quercetin pharmacokinetics in humans," Biopharmaceutics \& Drug Disposition, vol. 29, no. 4, pp. 205-217, 2008.

[53] M. Harwood, B. Danielewska-Nikiel, J. F. Borzelleca, G. W. Flamm, G. M. Williams, and T. C. Lines, "A critical review of the data related to the safety of quercetin and lack of evidence of _in vivo_toxicity, including lack of genotoxic/carcinogenic properties," Food and Chemical Toxicology: An International Journal Published for the British Industrial Biological Research Association, vol. 45, no. 11, pp. 2179-2205, 2007.

[54] F. Zhou, J. Mei, K. Yuan, X. Han, H. Qiao, and T. Tang, "Isorhamnetin attenuates osteoarthritis by inhibiting osteoclastogenesis and protecting chondrocytes through modulating reactive oxygen species homeostasis," Journal of Cellular and Molecular Medicine, vol. 23, no. 6, pp. 4395-4407, 2019.

[55] S. Kim, C.-Y. Jin, C. Kim et al., "Isorhamnetin alleviates lipopolysaccharide-induced inflammatory responses in BV2 microglia by inactivating NF- $\kappa \mathrm{B}$, blocking the TLR4 pathway and reducing ROS generation," International Journal of Molecular Medicine, vol. 43, no. 2, pp. 682-692, 2019.

[56] G. Gong, Y. Guan, and Z. Zhang, "Isorhamnetin: a review of pharmacological effects," Biomedicine \& Pharmacotherapy = Biomedecine \& Pharmacotherapie, vol. 128, p. 110301, 2020.

[57] K. Lan, X. Jiang, and J. He, "Quantitative determination of isorhamnetin, quercetin and kaempferol in rat plasma by liquid chromatography with electrospray ionization tandem mass spectrometry and its application to the pharmacokinetic study of isorhamnetin," Rapid Communications in Mass Spectrometry: RCM, vol. 21, no. 2, pp. 112-120, 2007.

[58] M. C. Sabini, L. N. Cariddi, F. M. Escobar et al., "Evaluation of the cytotoxicity, genotoxicity and apoptotic induction of an aqueous extract of _Achyrocline satureioides_(Lam.) DC," Food and Chemical Toxicology: An International Journal Published for the British Industrial Biological Research Association, vol. 60, pp. 463-470, 2013.

[59] Z. Zhuang, G. Ye, and B. Huang, "Kaempferol alleviates the interleukin-1 $\beta$-induced inflammation in rat osteoarthritis chondrocytes via suppression of NF- $\kappa \mathrm{B}$," Medical Science monitor: International Medical Journal of Experimental and Clinical Research, vol. 23, pp. 3925-3931, 2017.

[60] X. Huang, Q. Pan, Z. Mao et al., "Kaempferol inhibits interleukin- $1 \beta$ stimulated matrix metalloproteinases by suppressing the MAPK-associated ERK and P38 signaling pathways," Molecular Medicine Reports, vol. 18, no. 3, 2018.

[61] X. Cheng, Y. Yang, H. Yang, Y. Wang, and G. Du, "Kaempferol alleviates LPS-induced neuroinflammation and BBB dysfunction in mice via inhibiting HMGB1 release and down-regulating TLR4/MyD88 pathway," International Immunopharmacology, vol. 56, pp. 29-35, 2018.

[62] M.-S. Tsai, Y.-H. Wang, Y.-Y. Lai et al., "Kaempferol protects against propacetamol-induced acute liver injury through CYP2E1 inactivation, UGT1A1 activation, and attenuation of oxidative stress, inflammation and apoptosis in mice," Toxicology Letters, vol. 290, pp. 97-109, 2018.
[63] J. Park and J. Choi, "Role of kaempferol to increase bioavailability and pharmacokinetics of nifedipine in rats," Chinese Journal of Natural Medicines, vol. 17, no. 9, pp. 690-697, 2019.

[64] V. Zabela, C. Sampath, M. Oufir, F. Moradi-Afrapoli, V. Butterweck, and M. Hamburger, "Pharmacokinetics of dietary kaempferol and its metabolite 4-hydroxyphenylacetic acid in rats," Fitoterapia, vol. 115, pp. 189-197, 2016.

[65] K. P. Devi, D. S. Malar, S. F. Nabavi et al., "Kaempferol and inflammation: from chemistry to medicine," Pharmacological research, vol. 99, pp. 1-10, 2015.

[66] Y. Jia, W. He, H. Zhang et al., "Morusin ameliorates IL-1 $\beta$ induced chondrocyte inflammation and osteoarthritis via NF- $\kappa \mathrm{B}$ signal pathway," Drug Design, Development and Therapy, vol. Volume 14, pp. 1227-1240, 2020.

[67] X. Shi, S. Yang, G. Zhang et al., “The different metabolism of morusin in various species and its potent inhibition against UDP-glucuronosyltransferase (UGT) and cytochrome p450 (CYP450) enzymes," Xenobiotica; The Fate of Foreign Compounds in Biological Systems, vol. 46, no. 5, pp. 467-476, 2016.

[68] N. Im, D. Lee, S. Lee, and G. Jeong, "Lupeol isolated from Sorbus commixta suppresses $1 \alpha, 25-(\mathrm{OH}) 2 \mathrm{D} 3$-mediated osteoclast differentiation and bone loss in vitro and in vivo," Journal of Natural Products, vol. 79, no. 2, pp. 412-420, 2016.

[69] M. Xu, X. Li, L. Song, C. Tao, J. Fang, and L. Tao, "Lupeol alleviates coxsackievirus B3-induced viral myocarditis in mice via downregulating toll-like receptor 4," The Journal of International Medical Research, vol. 48, no. 4, article 030006052091090, 2020.

[70] K. Liu, X. Zhang, L. Xie et al., "Lupeol and its derivatives as anticancer and anti-inflammatory agents: Molecular mechanisms and therapeutic efficacy," Pharmacological Research, vol. 164, p. 105373, 2021.

[71] M. Cháirez-Ramírez, J. Gallegos-Infante, M. Moreno-Jiménez, R. González-Laredo, and N. Rocha-Guzmán, "Absorption and distribution of lupeol in CD-1 mice evaluated by UPLC-APCI +-MS/MS," Biomedical Chromatography: BMC, vol. 33, no. 3, p. e4432, 2019.

[72] M. Saleem, "Lupeol, a novel anti-inflammatory and anticancer dietary triterpene," Cancer Letters., vol. 285, no. 2, pp. 109-115, 2009.

[73] D. Zhang, B. Huang, C. Xiong, and Z. Yue, "Pinocembrin inhibits matrix metalloproteinase expression in chondrocytes," IUBMB Life, vol. 67, no. 1, pp. 36-41, 2015.

[74] X. Lan, X. Han, Q. Li et al., "Pinocembrin protects hemorrhagic brain primarily by inhibiting toll-like receptor 4 and reducing M1 phenotype microglia," Brain, Behavior, and Immunity, vol. 61, pp. 326-339, 2017.

[75] X. Shen, Y. Liu, X. Luo, and Z. Yang, "Advances in biosynthesis, pharmacology, and pharmacokinetics of pinocembrin, a promising natural small-molecule drug," Molecules (Basel, Switzerland), vol. 24, no. 12, p. 2323, 2019.

[76] W.-W. Guo, F. Qiu, X.-Q. Chen, Y.-Y. Ba, X. Wang, and $\mathrm{X}$. Wu, “_In-vivo_absorption of pinocembrin-7-_O- $\beta$ - $-\mathrm{D}$ glucoside in rats and its _in-vitro_biotransformation," Scientific Reports, vol. 6, no. 1, 2016.

[77] P. Lepetsos, K. Papavassiliou, and A. Papavassiliou, "Redox and NF- $\kappa \mathrm{B}$ signaling in osteoarthritis," Free Radical Biology \& Medicine, vol. 132, pp. 90-100, 2019.

[78] S. Rigoglou and A. Papavassiliou, "The NF- $\kappa$ B signalling pathway in osteoarthritis," The International Journal of Biochemistry \& Cell Biology, vol. 45, no. 11, pp. 2580-2584, 2013. 
[79] V. B. Kraus, G. McDaniel, J. L. Huebner et al., "Direct _in vivo_evidence of activated macrophages in human osteoarthritis," Osteoarthritis and Cartilage., vol. 24, no. 9, pp. 1613-1621, 2016.

[80] Z. Yang, Q. Yin, J. Ma et al., "Screen the effective components of Lycopodii herba on rheumatoid arthritis with the aid of Spectrum-effect relationship and uncover its potential mechanism," Inflammation, vol. 43, no. 6, pp. 2087-2097, 2020.

[81] Q. Sun, M. He, M. Zhang et al., "Ursolic acid: a systematic review of its pharmacology, toxicity and rethink on its pharmacokinetics based on PK-PD model," Fitoterapia, vol. 147, p. 104735, 2020.

[82] M. Arnoriaga-Rodríguez, J. Mayneris-Perxachs, A. Burokas et al., "Gut bacterial ClpB-like gene function is associated with decreased body weight and a characteristic microbiota profile," Microbiome, vol. 8, no. 1, p. 59, 2020.

[83] W. Feng, H. Ao, C. Peng, and D. Yan, "Gut microbiota, a new frontier to understand traditional Chinese medicines," Pharmacological Research, vol. 142, pp. 176-191, 2019.

[84] J. Lin and M. Yamazaki, "Role of P-glycoprotein in pharmacokinetics: clinical implications," Clinical Pharmacokinetics, vol. 42, no. 1, pp. 59-98, 2003.

[85] S. Zhou, "Drugs behave as substrates, inhibitors and inducers of human cytochrome P450 3A4," Current Drug Metabolism., vol. 9, no. 4, pp. 310-322, 2008.

[86] C. Wang, L. Chen, C. Xu et al., “A comprehensive review for phytochemical, pharmacological, and biosynthesis studies onGlycyrrhizaspp," The American Journal of Chinese Medicine, vol. 48, no. 1, pp. 17-45, 2020.

[87] X. Wang, H. Zhang, L. Chen, L. Shan, G. Fan, and X. Gao, "Liquorice, a unique "guide drug" of traditional Chinese medicine: a review of its role in drug interactions," Journal of Ethnopharmacology, vol. 150, no. 3, pp. 781-790, 2013.

[88] H. Tang, Y.-j. Wu, F. Xiao et al., "Regulation of CP-25 on Pglycoprotein in synoviocytes of rats with adjuvant arthritis," Biomedicine \& Pharmacotherapy = Biomedecine \& Pharmacotherapie, vol. 119, p. 109432, 2019.

[89] W. Zhang, W. Robertson, J. Zhao, W. Chen, and J. Xu, "Emerging trend in the pharmacotherapy of osteoarthritis," Frontiers in Endocrinology, vol. 10, 2019.

[90] P. Manyike, E. Kharasch, T. Kalhorn, and J. Slattery, "Contribution of CYP2E1 and CYP3A to acetaminophen reactive metabolite formation," Clinical Pharmacology and Therapeutics, vol. 67, no. 3, pp. 275-282, 2000.

[91] S. V. Bava, C. N. Sreekanth, A. K. T. Thulasidasan et al., "Akt is upstream and MAPKs are downstream of NF- $\kappa$ B in paclitaxelinduced survival signaling events, which are down-regulated by curcumin contributing to their synergism," The International Journal of Biochemistry \& Cell Biology, vol. 43, no. 3, pp. 331-341, 2011.

[92] J. Luo, H. Kamata, and M. Karin, "IKK/NF-kappaB signaling: balancing life and death-a new approach to cancer therapy," The Journal of Clinical Investigation, vol. 115, no. 10, pp. 2625-2632, 2005.

[93] T. Zhang, G. Bai, Y. Han et al., “The method of quality marker research and quality evaluation of traditional Chinese medicine based on drug properties and effect characteristics," Phytomedicine, vol. 44, pp. 204-211, 2018. 